\title{
Hamilton Connectivity of Convex Polytopes with Applications to Their Detour Index
}

\author{
Sakander Hayat $\mathbb{D}^{1},{ }^{1}$ Asad Khan $\left(\mathbb{D},{ }^{2}\right.$ Suliman Khan $\mathbb{D D}^{1}$, and Jia-Bao Liu $\mathbb{D}^{3}$ \\ ${ }^{1}$ Faculty of Engineering Sciences, GIK Institute of Engineering Sciences and Technology, Topi, Swabi, \\ Khyber Pakhtunkhwa 23460, Pakistan \\ ${ }^{2}$ School of Computer Science and Cyber Engineering, Guangzhou University, Guangzhou 510006, China \\ ${ }^{3}$ School of Mathematics and Physics, Anhui Jianzhu University, Hefei, Anhui 230000, China \\ Correspondence should be addressed to Asad Khan; asad@gzhu.edu.cn and Jia-Bao Liu; liujiabaoad@163.com
}

Received 1 November 2020; Revised 21 November 2020; Accepted 30 November 2020; Published 25 January 2021

Academic Editor: M. Irfan Uddin

Copyright (c) 2021 Sakander Hayat et al. This is an open access article distributed under the Creative Commons Attribution License, which permits unrestricted use, distribution, and reproduction in any medium, provided the original work is properly cited.

\begin{abstract}
A connected graph is called Hamilton-connected if there exists a Hamiltonian path between any pair of its vertices. Determining whether a graph is Hamilton-connected is an NP-complete problem. Hamiltonian and Hamilton-connected graphs have diverse applications in computer science and electrical engineering. The detour index of a graph is defined to be the sum of lengths of detours between all the unordered pairs of vertices. The detour index has diverse applications in chemistry. Computing the detour index for a graph is also an NP-complete problem. In this paper, we study the Hamilton-connectivity of convex polytopes. We construct three infinite families of convex polytopes and show that they are Hamilton-connected. An infinite family of nonHamilton-connected convex polytopes is also constructed, which, in turn, shows that not all convex polytopes are Hamiltonconnected. By using Hamilton connectivity of these families of graphs, we compute exact analytical formulas of their detour index.
\end{abstract}

\section{Introduction and Preliminaries}

All graphs in this paper are simple, loopless, finite, and connected.

A cycle in a graph $G$ is called Hamiltonian if it travels all the vertices of $G$. Moreover, a path in $G$ is called Hamiltonian path if it traverses all the vertices of $G$. Not every graph contains a Hamiltonian cycle. For instance, any tree is an acyclic graph, so it cannot contain a Hamiltonian cycle. However, a tress may still contain a Hamiltonian path. A graph $G$ is called Hamiltonian if there exist a Hamiltonian cycle in it. By definition, any cycle graph or a clique graph are Hamiltonian. Furthermore, $G$ is called traceable if it contains a Hamiltonian path. Of course all Hamiltonian graphs are traceable. However, there exist graphs which are traceable but not Hamiltonian. The first nontrivial example which comes in mind immediately is the so-called Petersen graph which is traceable but not Hamiltonian.

A graph which contains a Hamiltonian path between every two vertices of $G$ is called Hamilton connected. They were introduced by Ore [1] in 1963. Frucht [2] studied a canonical representation of trivalent Hamiltonian graphs. A bipartite can never be a Hamilton-connected, since there cannot exist a Hamiltonian path between vertices of the same partite set. In that case, a bipartite graph is called Hamilton-laceable if there exist Hamiltonian paths between vertices of different partite sets. There is an extensive literature available on Hamiltoniancity and Hamilton-connectivity of graphs, see, for instance, [3-7].

Chartrand et al. [8] showed that the square of a block graph is Hamilton-connected. Thomasson [9] studied Hamilton-connected tournaments in graphs. Chang et al. [10] studied panconnectivity, fault-tolerant Hamiltonicity, and Hamiltonian-connectivity in alternating group graphs by considering them as interconnection networks. Kewen et al. [11] derived a sufficient condition for a graph to be Hamilton-connected. Zhou and Wang [12] proved certain sufficient conditions for a graph to be Hamilton-connected in terms of the edge number, the spectral radius, and the signless Laplacian spectral radius of the graph. Zhou et al. 
[13] calculated the Wiener and Harary indices of Hamiltonconnected graphs with large diameter. Wei et al. [14] derived some spectral analogues of Erdös' theorems for Hamiltonconnected graphs. Hung et al. [15] studied Hamilton-connectivity of alphabet grid graphs. Zhou et al. [16] extended a result of Fiedler and Nikiforov and derived signless Laplacian spectral conditions for Hamilton-connected graphs with large minimum degree. Recently, Shabbir et al. [17] studied Hamilton-connectivity in Teoplitz graphs.

By preserving the vertex-edge incidence relation in convex polytopes, their graphs are constructed. Bača [18-20] was the first researcher to consider these families of geometric graphs. In [20] (resp. [19]), Bača studied the problem of magic (resp. graceful and antigraceful) labeling of convex polytopes, whereas, in [18], the problem of face antimagic labeling of convex polytopes was studied. Miller et al. [21] studied the vertex-magic total labeling of convex polytopes. Imran et al. [22-24] computed the minimum metric dimension of various infinite families of convex polytopes. In particular, they showed that these infinite families of convex polytopes have constant metric dimension. Malik et al. [25] also constructed two infinite families of convex polytopes having constant metric dimension. Other closely related infinite families of graphs with constant metric dimension are studied in [26]. Kratica et al. [27] studied the strong metric dimension of certain infinite families of convex polytopes by constructing their doubly resolving sets. The fault-tolerant metric dimension (resp. mixed metric dimension) of convex polytopes was studied by Raza et al. [28] (resp. Raza et al. [29]). Metric dimension of other related families of graphs such as Cayley graph is studied by Vetrik and Abas [30,31]. The binary locating-dominating number of convex polytopes is studied by Simić et al. [32] and Raza et al. [33]. The open-locating-dominating number of certain convex polytopes has recently been studied by Savic et al. [34]. Liu et al. [35-39] studied application of various graph parameters in electrical networks and related systems.

For a graph $G$, let $\ell(x, y)$ be the length of a longest path (i.e., detour) between vertices $x$ and $y$ of $G$. The detour index [40] is defined to be the sum of lengths of all detours between unordered pairs of vertices in $G$. The detour index of a graph $G$ is usually denoted by $\omega(G)$ :

$$
\omega(G)=\sum_{\{x, y\} \subset V(G)} \ell(x, y) .
$$

The detour index has important applications in chemistry. Applications of this parameter in quantitative structure activity and property relationship models were put forward by Lukovits [41]. Besides giving further applications of the detour index, Trinajstić et al. [42] conducted a comparative analysis with the Wiener index in terms of applicability in correlating structure boiling point of organic compounds. Moreover, its further applications in predicting the normal boiling points of cyclic and acyclic alkanes were studied by Rücker and Rücker [43].

An algorithm for tracing the detour between two vertices in a graph was proposed by Lukovits and Razinger [44]. They also applied their algorithm in detecting detours and computing the detour index of graphs corresponding to fused bicyclic skeletons. Rücker and Rücker [43] and Trinajstić et al. [45] proposed certain computer methods to trace out detours and thus calculation of the detour index of graphs. It has already been shown in [46] that the problem of finding the detour index of a given graph is computationally NP-complete. Trinajstić et al. [45] also proposed a method of calculating the detour matrix of reasonably small sizes. Note that the detour index is equal to the sum of all the entries of the detour matrix dividing by two.

Mahmiani et al. [47] proposed the edge versions of the detour index and studied their mathematical properties. Zhou and Cai [48] proved some upper and lower bounds on the detour index of graphs. Qi and Zhou [49] studied minimum uncyclic graphs with respect to the detour index. $\mathrm{Du}$ [50] studied the minimum detour index of bicyclic graphs. Fang et al. [51] characterized the minimum detour index of some families of tricyclic graphs. Karbasioun et al. [52] studied the applications of the detour index in infinite families of nanostar dendrimers. Wu and Deng [53] computed the detour index for a chain of C20 fullerenes. Kaladevi [54] studied spectral properties of the detour index in relation with the Laplacian energy of graphs. Recently, Abdullah and Omar [55] introduced the restricted edge version of the detour index and studied it for some families of graphs.

We end this section with an important and well-known result bounding the detour index in terms of graph parameters.

Theorem 1 (see [56]). Let $G$ be a connected graph with $n \geq 3$ vertices. Then,

$$
(n-1)^{2} \leq \omega(G) \leq \frac{n(n-1)^{2}}{2},
$$

with left equality if and only if $G \cong S_{n}$ and with right equality if and only if $G$ is Hamilton-connected.

In this paper, we study the Hamilton connectivity of certain infinite families of convex polytopes. More precisely, we construct three infinite families of Hamilton-connected convex polytopes. Moreover, we construct an infinite family non-Hamilton-connected convex polytope. By using Hamilton-connectivity of these families of graphs, we compute exact formulas of their detour index.

\section{Hamilton Connectivity and the Detour Index of $R_{n}$}

In this section, we show that the $n$-dimensional infinite family of convex polytopes $R_{n}$ is Hamilton-connected. Afterwards, we use its Hamiltonconnectivity to find analytical exact expression of the detour index of the graph $R_{n}$.

For $n \geq 5$, the graph of convex polytope $R_{n}$ is defined in [20]. It has the vertex set

$$
V\left(R_{n}\right)=\left\{x_{j} ; y_{j} ; z_{j}: 1 \leq j \leq n\right\},
$$

and edge set 


$$
\begin{aligned}
E\left(R_{n}\right)= & \left\{\left(x_{j}, x_{j+1}\right) ;\left(x_{j}, y_{j}\right) ;\left(x_{j+1}, y_{j}\right) ;\left(y_{j}, y_{j+1}\right) ;\right. \\
& \left.\cdot\left(y_{j}, z_{j}\right) ;\left(z_{j}, z_{j+1}\right): 1 \leq j \leq n\right\} .
\end{aligned}
$$

By convention, we suppose that $x_{n+1}=x_{n}, y_{n+1}=y_{n}$, and $z_{n+1}=z_{n}$. See Figure 1 to view the $n$-dimensional convex polytope $R_{n}$.

For a positive integer $v \in \mathbb{Z}^{+}$, we write $v \mid 2$ (resp. $v \nmid 2$ ) if $v$ is even (resp. odd).

Theorem 2. The n-dimensional convex polytope $R_{n}$, with $n \geq 4$, is Hamilton-connected.
Proof. We prove this result by definition. For this, we have to show that there exists a Hamiltonian path between any pair of vertices of $R_{n}$.

Let $P_{H}(u, v)$ be a Hamiltonian path between vertices $u$ and $v$ in $R_{n}$. Let $V\left(R_{n}\right)=Z \cup Y \cup X$ such that $Z=\left\{z_{1}, z_{2}, \ldots, z_{n}\right\}, \quad Y=\left\{y_{1}, y_{2} \ldots y_{n}\right\}, \quad$ and $X=\left\{x_{1}, x_{2}, \ldots, x_{n}\right\}$, see, Figure 1 .

Case 1: $u=z_{1}$ and $v=z_{i}, 2 \leq i \leq n$.

Case 1.1: $2 \leq i \leq n-1$ :

$P_{H}(u, v): u=z_{1} \sim\left\{z_{n-j}: 0 \leq j \leq n-i-1\right\} \sim\left\{y_{i-j+1}: 0 \leq j \leq i-2\right\} \sim\left\{x_{j}: 3 \leq j \leq i+1\right\} \sim\left\{x_{j} y_{j}: i+2 \leq j \leq n\right\} \sim x_{1} y_{1} x_{2} y_{2}$

$$
\sim\left\{z_{j}: 2 \leq j \leq i\right\}=v \text {. }
$$

Case 1.2: $i=n$ :

$P_{H}(u, v): u=z_{1} y_{1} \sim\left\{x_{j}: 1 \leq j \leq n\right\} \sim\left\{y_{n-j}: 0 \leq j \leq n-2\right\}$

$$
\sim\left\{z_{j}: 2 \leq j \leq n\right\}=v \text {. }
$$

Case 2: $u=z_{1}$ and $v=y_{i}, 1 \leq i \leq n$.

Case 2.1: $1 \leq i \leq n-1$ :

$P_{H}(u, v): u=z_{1} \sim\left\{z_{j}: 2 \leq j \leq n\right\} \sim\left\{y_{n-j}: 0 \leq j \leq n-i-1\right\}$

$$
\sim\left\{x_{j}: i+1 \leq j \leq n\right\} \sim\left\{x_{j} y_{j}: 1 \leq j \leq i\right\}=v .
$$

Case 2.2: $i=n$ :

$$
\begin{aligned}
P_{H}(u, v): u= & z_{1} y_{1} \sim\left\{x_{j}: 1 \leq j \leq n\right\} \sim\left\{y_{n-j}: 1 \leq j \leq n-2\right\} \\
& \sim\left\{z_{j}: 2 \leq j \leq n\right\} \sim y_{n}=v .
\end{aligned}
$$

Case 3: $u=z_{1}$ and $v=x_{i}, 1 \leq i \leq n$.

Case 3.1: $i=1$ :

$$
\begin{aligned}
P_{H}(u, v): u= & z_{1} \sim\left\{z_{j}: 2 \leq j \leq n\right\} \sim\left\{y_{n-j}: 0 \leq j \leq n-1\right\} \\
& \sim\left\{x_{j}: 2 \leq j \leq n\right\} \sim x_{1}=v .
\end{aligned}
$$

Case 3.2: $2 \leq i \leq n$ :

$$
\begin{aligned}
P_{H}(u, v): u= & z_{1} \sim\left\{z_{j}: 2 \leq j \leq n\right\} \sim\left\{y_{n-j}: 0 \leq j \leq n-i\right\} \\
& \sim\left\{y_{i-j} x_{i-j}: 1 \leq j \leq i-1\right\} \sim\left\{x_{n-j}: 0 \leq j \leq n-i\right\}=v .
\end{aligned}
$$

Case 4: $u=y_{1}$ and $v=z_{i}, 1 \leq i \leq n$.

Case 4.1: $i=1$ :

$$
P_{H}(u, v): u=y_{1} \sim\left\{x_{j}: 1 \leq j \leq n\right\} \sim\left\{y_{n-j}: 0 \leq j \leq n-2\right\}
$$$$
\sim\left\{z_{j}: 2 \leq j \leq n\right\} \sim z_{1}=v .
$$

Case 4.2: $2 \leq i \leq n-1$ :

$$
\begin{aligned}
P_{H}(u, v): u= & y_{1} x_{1} \sim\left\{x_{j} y_{j}: 2 \leq j \leq i\right\} \sim\left\{x_{j}: i+1 \leq j \leq n\right\} \\
& \sim\left\{y_{n-j}: 0 \leq j \leq n-i-1\right\} \sim\left\{z_{j}: i+1 \leq j \leq n\right\} \\
& \sim\left\{z_{j}: 1 \leq j \leq i\right\}=v .
\end{aligned}
$$

Case 4.3: $i=n$ :

$$
\begin{aligned}
P_{H}(u, v): u= & y_{1} \sim\left\{z_{j}: 1 \leq j \leq n-1\right\} \sim\left\{y_{n-j}: 1 \leq j \leq n-2\right\} \\
& \sim\left\{x_{j}: 2 \leq j \leq n\right\} \sim x_{1} y_{n} z_{n}=v .
\end{aligned}
$$




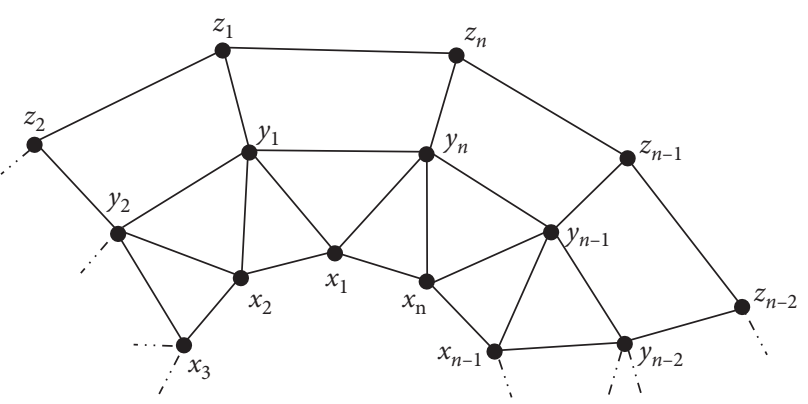

Figure 1: The $n$-dimensional convex polytope $R_{n}$.

Case 5: $u=y_{1}$ and $v=y_{i}, 2 \leq i \leq n$.

Case 5.1: $i=2$ :

$$
\begin{aligned}
P_{H}(u, v): u= & y_{1} \sim\left\{x_{j}: 1 \leq j \leq n\right\} \sim\left\{y_{n-j}: 0 \leq j \leq n-3\right\} \\
& \sim\left\{z_{j}: 3 \leq j \leq n\right\} \sim z_{1} z_{2} y_{2}=v .
\end{aligned}
$$

Case 5.2: $3 \leq i \leq n-1$ :

$$
\begin{aligned}
P_{H}(u, v): u= & y_{1} x_{1} \sim\left\{x_{j} y_{j}: 2 \leq j \leq i-1\right\} \sim\left\{x_{j}: i \leq j \leq n\right\} \\
& \sim\left\{y_{n-j}: 0 \leq j \leq n-i-1\right\} \sim\left\{z_{j}: i+1 \leq j \leq n\right\} \\
& \sim\left\{z_{j}: 1 \leq j \leq i\right\}=v .
\end{aligned}
$$

Case 5.3: $i=n$ :

$$
\begin{aligned}
P_{H}(u, v): u= & y_{1} z_{1} \sim\left\{z_{n-j}: 0 \leq j \leq n-2\right\} \\
& \sim\left\{y_{j}: 2 \leq j \leq n-1\right\} \sim\left\{x_{n-j}: 0 \leq j \leq n-1\right\} \\
& \sim y_{n}=v .
\end{aligned}
$$

Case 6: $u=y_{1}$ and $v=x_{i}, 1 \leq i \leq n$.

Case 6.1: $i=1,2$ :

$$
\begin{aligned}
P_{H}(u, v): u= & y_{1} \sim\left\{z_{j}: 1 \leq j \leq n\right\} \sim\left\{y_{n-j}: 0 \leq j \leq n-2\right\} \\
& \sim\left\{x_{j}: i+1 \leq j \leq n\right\} \sim\left\{x_{j}: 1 \leq j \leq i\right\}=v .
\end{aligned}
$$

Case 6.2: $3 \leq i \leq n-1$ :

$$
\begin{aligned}
P_{H}(u, v): u= & y_{1} \sim\left\{z_{j}: 1 \leq j \leq n\right\} \sim\left\{y_{n-j}: 0 \leq j \leq n-i\right\} \\
& \sim\left\{x_{j}: i+1 \leq j \leq n\right\} \sim x_{1} \sim\left\{x_{j} y_{j}: 2 \leq j \leq i-1\right\} \\
& \sim x_{i}=v .
\end{aligned}
$$

Case 6.3: $i=n$ :

$$
\begin{aligned}
P_{H}(u, v): u= & y_{1} x_{1} y_{n} z_{n} \sim\left\{z_{j}: 1 \leq j \leq n-1\right\} \\
& \sim\left\{y_{n-j}: 1 \leq j \leq n-2\right\} \sim\left\{x_{j}: 2 \leq j \leq n\right\}=v .
\end{aligned}
$$

Case 7: $u=x_{1}$ and $v=z_{i}, 1 \leq i \leq n$.

Case 7.1: $i=1$ :

$$
\begin{aligned}
P_{H}(u, v): u= & x_{1} \sim\left\{x_{n-j}: 0 \leq j \leq n-2\right\} \sim\left\{y_{j}: 1 \leq j \leq n\right\} \\
& \sim\left\{z_{n-j}: 0 \leq j \leq n-2\right\} \sim z_{1}=v .
\end{aligned}
$$

Case 7.2: $i=2$ :

$$
\begin{aligned}
P_{H}(u, v): u= & x_{1} \sim\left\{x_{j}: 2 \leq j \leq n\right\} \sim\left\{y_{n-j}: 0 \leq j \leq n-1\right\} \\
& \sim z_{1} \sim\left\{z_{n-j}: 0 \leq j \leq n-3\right\} \sim z_{2}=v .
\end{aligned}
$$

Case 7.3: $3 \leq i \leq n$ :

$$
\begin{aligned}
P_{H}(u, v): u= & \left\{x_{j} y_{j}: 1 \leq j \leq i-2\right\} \sim\left\{x_{j}: i-1 \leq j \leq n\right\} \\
& \sim\left\{y_{n-j}: 0 \leq j \leq n-i+1\right\} \\
& \sim\left\{z_{i-j}: 1 \leq j \leq i-1\right\} \sim\left\{z_{n-j}: 0 \leq j \leq n-i\right\}=v .
\end{aligned}
$$


Case 8: $u=x_{1}$ and $v=y_{i}, 1 \leq i \leq n$.

Case 8.1: $i=1$ :

$$
\begin{aligned}
P_{H}(u, v): u= & x_{1} \sim\left\{x_{j}: 2 \leq j \leq n\right\} \sim\left\{y_{n-j}: 0 \leq j \leq n-2\right\} \\
& \sim\left\{z_{j}: 2 \leq j \leq n\right\} \sim z_{1} y_{1}=v .
\end{aligned}
$$

Case 8.2: $2 \leq i \leq n-1$ :

$$
\begin{aligned}
P_{H}(u, v): u= & \left\{x_{j} y_{j}: 1 \leq j \leq i-1\right\} \sim\left\{x_{j}: i \leq j \leq n\right\} \\
& \sim\left\{y_{n-j}: 0 \leq j \leq n-i-1\right\} \\
& \sim\left\{z_{j}: i+1 \leq j \leq n\right\} \sim\left\{z_{j}: 1 \leq j \leq i\right\} \sim y_{i}=v .
\end{aligned}
$$

Case 8.3: $i=n$ :

$$
\begin{aligned}
P_{H}(u, v): u= & x_{1} \sim\left\{x_{j}: 2 \leq j \leq n\right\} \sim\left\{y_{n-j}: 1 \leq j \leq n-1\right\} \\
& \sim\left\{z_{j}: 1 \leq j \leq n\right\} \sim z_{n}=v .
\end{aligned}
$$

Case 9: $u=x_{1}$ and $v=x_{i}, 2 \leq i \leq n$.

Case 9.1: $2 \leq i \leq n-1$ :

$$
\begin{aligned}
P_{H}(u, v): u= & x_{1} \sim\left\{x_{n-j}: 0 \leq j \leq n-i-1\right\} \sim\left\{y_{j}: i \leq j \leq n\right\} \\
& \sim\left\{z_{n-j}: 0 \leq j \leq n-1\right\} \sim\left\{y_{j-1} x_{j}: 2 \leq j \leq i\right\}=v .
\end{aligned}
$$

Case 9.2: $i=n$ :

$$
\begin{aligned}
P_{H}(u, v): u= & x_{1} \sim\left\{x_{j}: 2 \leq j \leq n-1\right\} \sim\left\{y_{n-j}: 1 \leq j \leq n-1\right\} \\
& \sim\left\{z_{j}: 1 \leq j \leq n\right\} \sim y_{n} x_{n}=v .
\end{aligned}
$$

Existence of Hamiltonian path between any two vertices of the $n$-dimensional convex polytope $R_{n}$ completes the proof.

Using Theorems 1 and 2, the following proposition computes the detour index of of $R_{n}$.

Proposition 1. Let $G=R_{n}$, where $n \geq 4$. Then, the detour index of $G$ is

$$
\omega(G)=\frac{3 n(3 n-1)^{2}}{2} .
$$

Proof. The number of vertices in the graph $G$ is $3 n$. Replacing $3 n$ with $n$ in Theorem 1 shows the proposition.

\section{Hamilton Connectivity and the Detour Index of $S_{n}$}

In this section, we show that the $n$-dimensional infinite family of convex polytopes $S_{n}$ is Hamilton-connected. Then, we use its Hamilton connectivity to find analytical exact expression of the detour index of the graph $S_{n}$.

The graph of convex polytope $S_{n}$ (Figure 2) consists of $2 n$ 3 -sided faces, $2 n 4$-sided faces, and a pair of $n$-sided faces [57]. We have

$$
\begin{aligned}
& V\left(S_{n}\right)=\left\{w_{j} ; x_{j} ; y_{j} ; z_{j}: 1 \leq j \leq n\right\}, \\
& E\left(S_{n}\right)=\left\{w_{j} w_{j+1} ; x_{j} x_{j+1} ; y_{j} y_{j+1} ; z_{j} z_{j+1}: 1 \leq j \leq n\right\} \cup\left\{w_{j+1} x_{j} ; w_{j} x_{j} ; x_{j} y_{j} ; y_{j} z_{j}: 1 \leq j \leq n\right\} .
\end{aligned}
$$

See Figure 2 to view the $n$-dimensional convex polytope $S_{n}$

Next, we show the main result of this section.

Theorem 3. The n-dimensional convex polytope $S_{n}$, with $n \geq 4$, is Hamilton-connected.
Proof. We prove it by definition. This implies that we need to show the existence of Hamiltonian paths between any pair of vertices of $G$.

Let $P_{H}(u, v)$ be a Hamiltonian path between vertices $u$ and $v$ in $S_{n}$. By following the labeling of vertices exhibited in Figure 2, we show the existence of Hamiltonian paths between vertices of $S_{n}$ in a number of cases. 


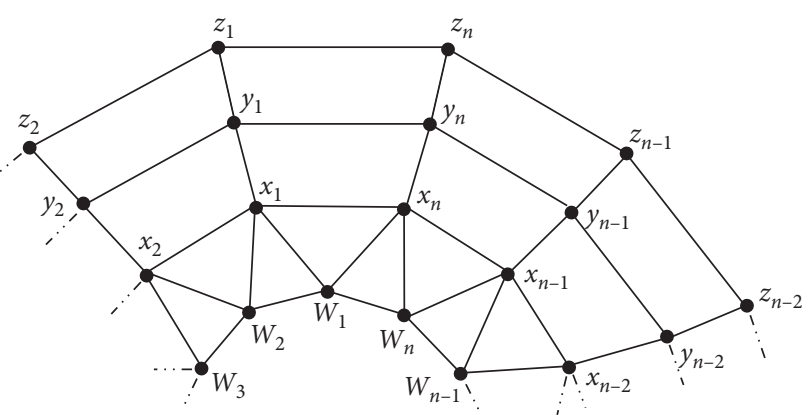

Figure 2: The $n$-dimensional convex polytope $S_{n}$.

Case 1: $u=z_{1}$ and $v=z_{i}, 2 \leq i \leq n$.

Case 1.1: $i \mid 2$ :

$$
\begin{gathered}
P_{H}(u, v): u=z_{1} y_{1} x_{1} w_{1} \sim\left\{w_{j-n} x_{j-n} y_{j-n} z_{j-n} z_{j-n+1} y_{j-n+1} x_{j-n+1} w_{j-n+1}: n+2 \leq j \leq\left(\frac{n+i}{2}\right)\right\} \sim\left\{w_{j}: i \leq j \leq n\right\} \\
\sim\left\{x_{n-j}: 0 \leq j \leq n-i\right\} \sim\left\{y_{j}: i \leq j \leq n\right\} \sim\left\{z_{n-j}: 0 \leq j \leq n-i\right\}=v .
\end{gathered}
$$

Case 1.2: i†2:

$$
\begin{aligned}
P_{H}(u, v): u= & z_{1} \sim\left\{z_{j}: 2 \leq j \leq i-1\right\} \sim\left\{y_{j}: i-1 \leq j \leq n-1\right\} \sim\left\{x_{n-j}: 1 \leq j \leq n-i+1\right\} \sim\left\{w_{j}: i-1 \leq j \leq n\right\} \\
& \sim x_{n} \sim\left\{w_{j}: 1 \leq j \leq i-2\right\} \sim\left\{x_{i-j} y_{i-j} y_{i-j-1} x_{i-j-1}: 2 \leq j \leq \frac{(i-1)}{2}\right\} \sim x_{1} y_{1} y_{n} \sim\left\{z_{n-j}: 0 \leq j \leq n-i\right\}=v .
\end{aligned}
$$

Case 2: $u z_{1}$ and $v y_{i}, 1 \leq i \leq n$ :

Case 2.1: $1 \leq i \leq n-1$ :

$$
\begin{aligned}
P_{H}(u, v): u= & z_{1} \sim\left\{z_{j}: 2 \leq j \leq n\right\} \sim\left\{y_{n-j}: 0 \leq j \leq n-i-1\right\} \sim\left\{x_{j}: i+1 \leq j \leq n\right\} \sim w_{1} \sim\left\{w_{n-j}: 0 \leq j \leq n-i-1\right\} \\
& \sim\left\{x_{i-j} w_{i-j}: 0 \leq j \leq i-2\right\} \sim x_{1} \sim\left\{y_{j}: 1 \leq j \leq i\right\}=v .
\end{aligned}
$$

Case 2.2: $i=n$ :

$P_{H}(u, v): u=z_{1} y_{1} x_{1} x_{n} w_{1} \sim\left\{w_{n-j}: 0 \leq j \leq n-2\right\} \sim\left\{x_{j}: 2 \leq j \leq n-1\right\} \sim\left\{y_{n-j}: 1 \leq j \leq n-2\right\} \sim\left\{z_{j}: 2 \leq j \leq n\right\} \sim y_{n}=v$.

Case 3: $u=z_{1}$ and $v=x_{i}, 1 \leq i \leq n$.

Case 3.1: $i=1$ : 
$P_{H}(u, v): u=z_{1} \sim\left\{z_{n-j}: 0 \leq j \leq n-2\right\} \sim y_{1} \sim\left\{y_{n-j}: 0 \leq j \leq n-3\right\} \sim\left\{x_{j}: 3 \leq j \leq n\right\} \sim w_{1} \sim\left\{w_{n-j}: 0 \leq j \leq n-2\right\} \sim x_{2} x_{1}=v$.

Case 3.2: $2 \leq i \leq n-1$ :

$$
\begin{aligned}
P_{H}(u, v): u= & z_{1} \sim\left\{z_{n-j}: 0 \leq j \leq n-2\right\} \sim\left\{y_{j}: 2 \leq j \leq n\right\} \sim y_{1} x_{1} \sim\left\{x_{n-j}: 0 \leq j \leq n-i-1\right\} \sim\left\{w_{j}: i+1 \leq j \leq n\right\} \\
& \sim w_{1} \sim\left\{w_{j} x_{j}: 2 \leq j \leq i\right\}=v .
\end{aligned}
$$

Case 3.3: $i=n$ :

$P_{H}(u, v): u=\left\{z_{j}: 1 \leq j \leq n\right\} \sim\left\{y_{n-j}: 0 \leq j \leq n-1\right\} \sim\left\{x_{j}: 1 \leq j \leq n-1\right\} \sim\left\{w_{n-j}: 0 \leq j \leq n-1\right\} \sim x_{n}=v$.

Case 4: $u=z_{1}$ and $v=w_{i}, 1 \leq i \leq n$.

Case 4.1: $i=1$ :

$$
P_{H}(u, v): u=\left\{z_{j}: 1 \leq j \leq n\right\} \sim\left\{y_{n-j}: 0 \leq j \leq n-1\right\} \sim\left\{x_{j}: 1 \leq j \leq n\right\} \sim\left\{w_{n-j}: 0 \leq j \leq n-1\right\}=v .
$$

Case 4.2: $2 \leq i \leq n-1$ :

$$
\begin{aligned}
P_{H}(u, v): u= & \left\{z_{j}: 1 \leq j \leq n\right\} \sim y_{n} \sim\left\{y_{j}: 1 \leq j \leq n-1\right\} \sim\left\{x_{n-j}: 1 \leq j \leq n-i\right\} \sim\left\{w_{j}: i+1 \leq j \leq n\right\} \\
& \sim w_{1} x_{n} \sim\left\{x_{j-1} w_{j}: 2 \leq j \leq i\right\}=v .
\end{aligned}
$$

Case 4.3: $i=n$ :

$$
P_{H}(u, v): u=\left\{z_{j}: 1 \leq j \leq n\right\} \sim\left\{y_{n-j}: 0 \leq j \leq n-1\right\} \sim\left\{x_{j}: 1 \leq j \leq n\right\} \sim\left\{w_{j}: 1 \leq j \leq n\right\}=v .
$$

Case 5: $u=y_{1}$ and $v=z_{i}, 1 \leq i \leq n$.

Case 5.1: $i=1$ :

$$
P_{H}(u, v): u=\left\{y_{j}: 1 \leq j \leq n-1\right\} \sim\left\{x_{n-j}: 1 \leq j \leq n-1\right\} \sim\left\{w_{j}: 1 \leq j \leq n\right\} \sim x_{n} y_{n} \sim\left\{z_{n-j}: 0 \leq j \leq n-1\right\}=v .
$$

Case 5.2: $2 \leq i \leq n-1$ : 


$$
\begin{aligned}
P_{H}(u, v): u= & \left\{y_{j}: 1 \leq j \leq i\right\} \sim\left\{x_{i-j} w_{i-j}: 0 \leq j \leq i-1\right\} \sim\left\{w_{n-j}: 0 \leq j \leq n-i-1\right\} \sim\left\{x_{j}: i+1 \leq j \leq n\right\} \\
& \sim\left\{y_{n-j}: 0 \leq j \leq n-i-1\right\} \sim\left\{z_{j}: i+1 \leq j \leq n\right\} \\
& \left\{z_{j}: 1 \leq j \leq i\right\}=v .
\end{aligned}
$$

Case 5.3: $i=n$ :

$P_{H}(u, v): u=y_{1} \sim\left\{z_{j}: 1 \leq j \leq n-1\right\} \sim\left\{y_{n-j}: 1 \leq j \leq n-2\right\} \sim\left\{x_{j}: 2 \leq j \leq n-1\right\} \sim\left\{w_{n-j}: 0 \leq j \leq n-1\right\} \sim x_{1} x_{n} y_{n} z_{n}=v$.

Case 6: $u=y_{1}$ and $v=y_{i}, 2 \leq i \leq n$.

Case 6.1: $2 \leq i \leq n-1$ :

$$
\begin{aligned}
P_{H}(u, v): u= & y_{1} \sim\left\{z_{j}: 1 \leq j \leq n\right\} \sim\left\{y_{n-j}: 0 \leq j \leq n-i-1\right\} \sim\left\{x_{i-j+1}: 0 \leq j \leq i-2\right\} \sim\left\{w_{j}: 3 \leq j \leq i+1\right\} \\
& \sim\left\{w_{j} x_{j}: i+2 \leq j \leq n\right\} \sim w_{1} x_{1} w_{2} x_{2} \sim\left\{y_{j}: 2 \leq j \leq i\right\}=v .
\end{aligned}
$$

Case 6.2: $i=n$ :

$P_{H}(u, v): u=y_{1} z_{1} \sim\left\{z_{n-j}: 0 \leq j \leq n-2\right\} \sim\left\{y_{j}: 2 \leq j \leq n-1\right\} \sim\left\{x_{n-j}: 1 \leq j \leq n-1\right\} \sim\left\{w_{j}: 1 \leq j \leq n\right\} \sim x_{n} y_{n}=v$.

Case 7: $u=y_{1}$ and $v=x_{i}, 1 \leq i \leq n$.

Case 7.1: $1 \leq i \leq n-1$ :

$$
\begin{aligned}
P_{H}(u, v): u= & y_{1} z_{1} \sim\left\{z_{n-j}: 0 \leq j \leq n-2\right\} \sim\left\{y_{j}: 2 \leq j \leq n\right\} \sim\left\{x_{n-j}: 0 \leq j \leq n-i-1\right\} \sim\left\{w_{j}: i+1 \leq j \leq n\right\} \\
& \sim\left\{w_{j} x_{j}: 1 \leq j \leq i\right\}=v .
\end{aligned}
$$

Case 7.2: $i=n$ :

$P_{H}(u, v): u=y_{1} \sim\left\{z_{j}: 1 \leq j \leq n\right\} \sim\left\{y_{n-j}: 0 \leq j \leq n-2\right\} \sim\left\{x_{j}: 2 \leq j \leq n-1\right\} \sim\left\{w_{n-j}: 0 \leq j \leq n-1\right\} \sim x_{1} x_{n}=v$.

Case 8: $u=y_{1}$ and $v=w_{i}, 1 \leq i \leq n$.

Case 8.1: $i=1$ :

$$
P_{H}(u, v): u=y_{1} z_{1} \sim\left\{z_{n-j}: 0 \leq j \leq n-2\right\} \sim\left\{y_{j}: 2 \leq j \leq n\right\} \sim\left\{x_{n-j}: 0 \leq j \leq n-1\right\} \sim\left\{w_{j}: 2 \leq j \leq n\right\} \sim w_{1}=v .
$$




$$
\begin{aligned}
P_{H}(u, v): u= & y_{1} z_{1} \sim\left\{z_{n-j}: 0 \leq j \leq n-2\right\} \sim\left\{y_{j}: 2 \leq j \leq n\right\} \sim\left\{x_{n-j}: 0 \leq j \leq n-i\right\} \sim\left\{x_{i-j} w_{i-j}: 1 \leq j \leq i-1\right\} \\
& \sim\left\{w_{n-j}: 0 \leq j \leq n-i\right\}=v .
\end{aligned}
$$

Case 9: $u=x_{1}$ and $v=z_{i}, 1 \leq i \leq n$.

Case 9.1: $i=1$ :

$P_{H}(u, v): u=x_{1} \sim\left\{y_{j}: 1 \leq j \leq n-1\right\} \sim\left\{x_{n-j}: 1 \leq j \leq n-2\right\} \sim\left\{w_{j}: 2 \leq j \leq n\right\} \sim x_{1} x_{n} y_{n} \sim\left\{z_{n-j}: 0 \leq j \leq n-1\right\}=v$.

Case 9.2: $2 \leq i \leq n-1$ :

$$
\begin{aligned}
P_{H}(u, v): u= & x_{1} y_{1} z_{1} \sim\left\{z_{n-j}: 0 \leq j \leq n-i-1\right\} \sim\left\{y_{j}: i+1 \leq j \leq n\right\} \sim\left\{x_{n-j}: 0 \leq j \leq n-i-1\right\} \sim\left\{w_{j}: i+1 \leq j \leq n\right\} \\
& \sim w_{1} \sim\left\{w_{j} x_{j}: 2 \leq j \leq i\right\} \sim\left\{y_{i-j}: 0 \leq j \leq i-2\right\} \sim\left\{z_{j}: 2 \leq j \leq i\right\}=v .
\end{aligned}
$$

Case 9.3: $i=n$ :

$$
P_{H}(u, v): u=x_{1} w_{1} \sim\left\{w_{n-j}: 0 \leq j \leq n-2\right\} \sim\left\{x_{j}: 2 \leq j \leq n\right\} \sim\left\{y_{n-j}: 0 \leq j \leq n-1\right\} \sim\left\{z_{j}: 1 \leq j \leq n\right\}=v .
$$

Case 10: $u=x_{1}$ and $v=y_{i}, 1 \leq i \leq n$.

Case 10.1: $i=1$ :

$$
P_{H}(u, v): u=x_{1} \sim\left\{w_{j}: 1 \leq j \leq n\right\} \sim\left\{x_{n-j}: 0 \leq j \leq n-2\right\} \sim\left\{y_{j}: 2 \leq j \leq n\right\} \sim\left\{z_{n-j}: 0 \leq j \leq n-1\right\} \sim y_{1}=v .
$$

Case 10.2: $2 \leq i \leq n-1$ :

$$
\begin{aligned}
P_{H}(u, v): u= & x_{1} y_{1} \sim\left\{z_{j}: 1 \leq j \leq n\right\} \sim\left\{y_{n-j}: 0 \leq j \leq n-i-1\right\} \sim\left\{x_{j} w_{j}: i+1 \leq j \leq n\right\} \sim\left\{w_{j}: 1 \leq j \leq i\right\} \\
& \sim\left\{x_{i-j}: 0 \leq j \leq i-2\right\} \sim\left\{y_{j}: 2 \leq j \leq i\right\}=v .
\end{aligned}
$$

Case 10.3: $i=n$ :

$P_{H}(u, v): u=x_{1} y_{1} z_{1} \sim\left\{z_{n-j}: 0 \leq j \leq n-2\right\} \sim\left\{y_{j}: 2 \leq j \leq n-1\right\} \sim\left\{x_{n-j}: 1 \leq j \leq n-2\right\} \sim\left\{w_{j}: 2 \leq j \leq n\right\} \sim w_{1} x_{n} y_{n}=v$.

Case 11: $u=x_{1}$ and $v=x_{i}, 2 \leq i \leq n$.

Case 11.1: $2 \leq i \leq n-1$ : 


$$
\begin{aligned}
P_{H}(u, v): u= & x_{1} y_{1} z_{1} \sim\left\{z_{n-j}: 0 \leq j \leq n-2\right\} \sim\left\{y_{j}: 2 \leq j \leq n\right\} \sim\left\{x_{n-j}: 0 \leq j \leq n-i-1\right\} \sim\left\{w_{j}: i+1 \leq j \leq n\right\} \\
& \sim w_{1} \sim\left\{w_{j} x_{j}: 2 \leq j \leq i\right\}=v .
\end{aligned}
$$

Case 11.2: $i=n$ :

$P_{H}(u, v): u=x_{1} y_{1} \sim\left\{z_{j}: 1 \leq j \leq n\right\} \sim\left\{y_{n-j}: 0 \leq j \leq n-2\right\} \sim\left\{x_{j}: 2 \leq j \leq n-1\right\} \sim\left\{w_{n-j}: 0 \leq j \leq n-1\right\} \sim x_{n}=v$.

Case 12: $u=x_{1}$ and $v=w_{i}, 1 \leq i \leq n$.

Case 12.1: $i=1$ :

$P_{H}(u, v): u=x_{1} y_{1} z_{1} \sim\left\{z_{n-j}: 0 \leq j \leq n-2\right\} \sim\left\{y_{j}: 2 \leq j \leq n\right\} \sim\left\{x_{n-j}: 0 \leq j \leq n-2\right\} \sim\left\{w_{j}: 2 \leq j \leq n\right\} \sim w_{1}=v$.

Case 12.2: $2 \leq i \leq n-1$ :

$$
\begin{aligned}
& P_{H}(u, v): u= x_{1} y_{1} z_{1} \sim\left\{z_{n-j}: 0 \leq j \leq n-2\right\} \sim\left\{y_{j}: 2 \leq j \leq n\right\} \sim\left\{x_{n-j}: 0 \leq j \leq n-i\right\} \sim\left\{w_{j}: i+1 \leq j \leq n\right\} \\
& \sim w_{1} \sim\left\{w_{j} x_{j}: 2 \leq j \leq i-1\right\} \sim w_{i}=v .
\end{aligned}
$$

Case 12.3: $i=n$ :

$$
P_{H}(u, v): u=\left\{x_{j}: 1 \leq j \leq n-1\right\} \sim\left\{y_{n-j}: 0 \leq j \leq n-1\right\} \sim\left\{z_{j}: 1 \leq j \leq n\right\} \sim y_{n} x_{n} \sim\left\{w_{j}: 1 \leq j \leq n\right\}=v
$$

Case 13: $u=w_{1}$ and $v=z_{i}, 1 \leq i \leq n$.

Case 13.1: $i=1$ :

$$
P_{H}(u, v): u=\left\{w_{j}: 1 \leq j \leq n\right\} \sim\left\{x_{n-j}: 0 \leq j \leq n-1\right\} \sim\left\{y_{j}: 1 \leq j \leq n\right\} \sim\left\{z_{n-j}: 0 \leq j \leq n-1\right\}=v .
$$

Case 13.2: $2 \leq i \leq n$ :

$$
\begin{aligned}
P_{H}(u, v): u= & \left\{w_{j} x_{j}: 1 \leq j \leq i-1\right\} \sim\left\{w_{j}: i \leq j \leq n\right\} \sim\left\{x_{n-j}: 0 \leq j \leq n-i\right\} \sim\left\{y_{j}: i \leq j \leq n\right\} \\
& \sim\left\{y_{j}: 1 \leq j \leq i-1\right\} \sim\left\{z_{i-j}: 1 \leq j \leq i-1\right\} \sim\left\{z_{n-j}: 0 \leq j \leq n-i\right\}=v .
\end{aligned}
$$

Case 14: $u=w_{1}$ and $v=y_{i}, 1 \leq i \leq n$.

Case 14.1: $i=1$ : 


$$
\begin{aligned}
P_{H}(u, v): u= & w_{1} \sim\left\{w_{n-j}: 0 \leq j \leq n-2\right\} \sim\left\{x_{j}: 1 \leq j \leq n\right\} \sim\left\{y_{n-j}: 0 \leq j \leq n-2\right\} \sim\left\{z_{j}: 2 \leq j \leq n\right\} \\
& \sim z_{1} y_{1}=v .
\end{aligned}
$$

Case 14.2: $2 \leq i \leq n-1$ :

$$
\begin{aligned}
P_{H}(u, v): u= & \left\{w_{j} x_{j}: 1 \leq j \leq i\right\} \sim\left\{w_{j}: i+1 \leq j \leq n\right\} \\
& \sim\left\{x_{n-j}: 0 \leq j \leq n-i-1\right\} \sim\left\{y_{j}: 1 \leq j \leq i-1\right\} \\
& \sim\left\{z_{i-j}: 1 \leq j \leq i-1\right\} \sim\left\{z_{n-j}: 0 \leq j \leq n-i\right\} \\
& \sim y_{i}=v .
\end{aligned}
$$

Case 14.3: $i=n$ :

$$
\begin{aligned}
P_{H}(u, v): u= & \left\{w_{j}: 1 \leq j \leq n\right\} \sim\left\{x_{n-j}: 0 \leq j \leq n-1\right\} \\
& \sim\left\{y_{j}: 1 \leq j \leq n-1\right\} \sim\left\{z_{n-j}: 1 \leq j \leq n-1\right\} \\
& \sim z_{n} y_{n}=v .
\end{aligned}
$$

Case 15: $u=w_{1}$ and $v=x_{i}, 1 \leq i \leq n$.

Case 15.1: $i=1$ :

$$
\begin{aligned}
P_{H}(u, v): u= & \left\{w_{j}: 1 \leq j \leq n\right\} \sim\left\{x_{n-j}: 0 \leq j \leq n-2\right\} \\
& \sim\left\{y_{j}: 2 \leq j \leq n\right\} \sim\left\{z_{n-j}: 0 \leq j \leq n-1\right\} \\
& \sim y_{1} x_{1}=v .
\end{aligned}
$$

Case 15.2: $2 \leq i \leq n-1$ :

$$
\begin{aligned}
P_{H}(u, v): u= & \left\{w_{j} x_{j}: 1 \leq j \leq i-1\right\} \sim\left\{w_{j}: i \leq j \leq n\right\} \\
& \sim\left\{x_{n-j}: 0 \leq j \leq n-i-1\right\} \sim\left\{y_{j}: i+1 \leq j \leq n\right\} \\
& \sim\left\{y_{j}: 1 \leq j \leq i-1\right\} \\
& \sim\left\{z_{i-j}: 1 \leq j \leq i-1\right\}\left\{z_{n-j}: 0 \leq j \leq n-i\right\} \\
& \sim y_{i} x_{i}=v .
\end{aligned}
$$

Case 15.3: $i=n$ :

$$
\begin{aligned}
P_{H}(u, v): u= & w_{1} \sim\left\{w_{n-j}: 0 \leq j \leq n-2\right\} \\
& \sim\left\{x_{j}: 1 \leq j \leq n-1\right\} \sim\left\{y_{n-j}: 1 \leq j \leq n-1\right\} \\
& \sim\left\{z_{j}: 1 \leq j \leq n\right\} \sim y_{n} x_{n}=v .
\end{aligned}
$$

Case 16: $u=w_{1}$ and $v=w_{i}, 2 \leq i \leq n$.

Case 16.1: $2 \leq i \leq n-1$

$$
\begin{aligned}
P_{H}(u, v): u= & w_{1} \sim\left\{w_{n-j}: 0 \leq j \leq n-i-1\right\} \sim\left\{x_{j}: i \leq j \leq n\right\} \sim\left\{y_{n-j}: 0 \leq j \leq n-2\right\} \sim\left\{z_{j}: 2 \leq j \leq n\right\} \sim z_{1} y_{1} x_{1} \\
& \sim\left\{w_{j} x_{j}: 2 \leq j \leq i-1\right\} \sim w_{i}=v .
\end{aligned}
$$

Case 16.2: $i=n$ :

$$
P_{H}(u, v): u=\left\{w_{j}: 1 \leq j \leq n-1\right\} \sim\left\{x_{n-j}: 1 \leq j \leq n-1\right\} \sim\left\{y_{j}: 1 \leq j \leq n-1\right\} \sim\left\{z_{n-j}: 1 \leq j \leq n-1\right\} \sim z_{n} y_{n} x_{n} w_{n}=v .
$$

Existence of Hamiltonian paths between any two vertices of the $n$-dimensional convex polytope $S_{n}$ completes the proof.

Using Theorems 1 and 3, the following proposition computes the detour index of $S_{n}$.

Proposition 2. Let $G=S_{n}$, where $n \geq 3$. Then, the detour index of $G$ is

$$
\omega(G)=\frac{4 n(4 n-1)^{2}}{2} .
$$

Proof. The number of vertices in graph $G$ is $4 n$. Replacing $4 n$ with $n$ in Theorem 1 completes the proof.

\section{Hamilton Connectivity and the Detour Index of $K_{n}$}

This section shows that the $n$-dimensional convex polytope $K_{n}$ is Hamilton-connected. Its Hamilton connectivity is then used to calculate exact analytical formula of the detour index of $K_{n}$. 
Malik et al. [25] introduced the family of $n$-dimensional convex polytope $K_{n}$. We have

$$
\begin{aligned}
& V\left(K_{n}\right)=\left\{v_{i} ; w_{j} ; x_{j} ; y_{j} ; z_{j}: 1 \leq j \leq n\right\}, \\
& E\left(K_{n}\right)=\left\{v_{j} v_{j+1} ; w_{j} w_{j+1} ; x_{j} x_{j+1} ; y_{j} y_{j+1} ; z_{j} z_{j+1}: 1 \leq j \leq n\right\} \cup\left\{v_{j} y_{j} ; v_{j} w_{j+1} ; w_{j} x_{j} ; x_{j} y_{j} ; x_{j} y_{j+1} ; y_{j} z_{j}: 1 \leq j \leq n\right\} .
\end{aligned}
$$
$K_{n}$.

See Figure 3 to view the $n$-dimensional convex polytope

Theorem 4. The graph $n$-dimensional convex polytope $K_{n}$, with $n \geq 5$, is Hamilton-connected.

Proof. We prove this result by definition. For this, we have to show that there exists a Hamiltonian path between any pair of vertices of $K_{n}$.

Let $P_{H}\left(u^{\prime}, v^{\prime}\right)$ be a Hamiltonian path between vertices $u^{\prime}$ and $v^{\prime}$ in $K_{n}$. Let $V\left(R_{n}\right)=Z \cup Y \cup X \cup V$ such that
$Z=\left\{z_{1}, z_{2}, \ldots, z_{n}\right\}, \quad Y=\left\{y_{1}, y_{2} \ldots y_{n}\right\}, \quad X=\left\{x_{1}, x_{2}\right.$, $\left.\ldots, x_{n}\right\}$, and $V=\left\{v_{1}, v_{2}, \ldots, v_{n}\right\}$. We divide the construction of Hamiltonian paths in $K_{n}$ into a number of cases as follows.

Case 1: $u^{\prime}=z_{1}$ and $v^{\prime}=z_{i}, 2 \leq i \leq n$.

Case 1.1: $2 \leq i \leq n-1$ :

$$
\begin{gathered}
P_{H}\left(u^{\prime}, v^{\prime}\right): u^{\prime}=z_{1} \sim\left\{z_{n-j}: 0 \leq j \leq n-i-1\right\} \sim\left\{y_{j}: i+1 \leq j \leq n\right\} \sim\left\{x_{n-j}: 0 \leq j \leq n-i\right\} \sim\left\{y_{i-j} x_{i-j-1}: 0 \leq j \leq i-3\right\} \\
\sim\left\{w_{j}: 2 \leq j \leq n\right\} \sim\left\{v_{n-j}: 0 \leq j \leq n-1\right\} \sim w_{1} x_{1} y_{1} y_{2} \sim\left\{z_{j}: 2 \leq j \leq i\right\}=v^{\prime}
\end{gathered}
$$

Case 1.2: $i=n$ :

$$
\begin{aligned}
P_{H}\left(u^{\prime}, v^{\prime}\right): u^{\prime}= & \left\{z_{j}: 1 \leq j \leq n-1\right\} \sim\left\{y_{n-j}: 1 \leq j \leq n-1\right\} \sim\left\{x_{j}: 1 \leq j \leq n-1\right\} \sim\left\{w_{n-j}: 1 \leq j \leq n-1\right\} \\
& \sim\left\{v_{j}: 1 \leq j \leq n\right\} \sim w_{n} x_{n} y_{n} z_{n}=v^{\prime} .
\end{aligned}
$$

Case 2: $u^{\prime}=z_{1}$ and $v^{\prime}=y_{i}, 1 \leq i \leq n$.

Case 2.1: $1 \leq i \leq n-1$ :

$$
\begin{aligned}
P_{H}\left(u^{\prime}, v^{\prime}\right): u^{\prime}= & \left\{z_{j}: 1 \leq j \leq n\right\} \sim\left\{y_{n-j}: 0 \leq j \leq n-i-1\right\} \sim\left\{x_{j}: i+1 \leq j \leq n\right\} \sim\left\{w_{n-j}: 0 \leq j \leq n-i-1\right\} \\
& \sim\left\{v_{j}: i+1 \leq j \leq n\right\} \sim\left\{v_{j} w_{j}: 1 \leq j \leq i\right\} \sim\left\{x_{i-j}: 0 \leq j \leq i-1\right\} \sim\left\{y_{j}: 1 \leq j \leq i\right\}=v^{\prime} .
\end{aligned}
$$

Case 2.2: $i=n$ :

$$
\begin{aligned}
P_{H}\left(u^{\prime}, v^{\prime}\right): u^{\prime}= & z_{1} \sim\left\{z_{n-j}: 0 \leq j \leq n-2\right\} \sim\left\{y_{j}: 2 \leq j \leq n-1\right\} \sim\left\{x_{n-j}: 1 \leq j \leq n-2\right\} \sim\left\{w_{j}: 2 \leq j \leq n\right\} \\
& \sim\left\{v_{n-j}: 0 \leq j \leq n-1\right\} \sim w_{1} x_{1} y_{1} x_{n} y_{n}=v^{\prime} .
\end{aligned}
$$

Case 3: $u^{\prime}=z_{1}$ and $v^{\prime}=x_{i}, 1 \leq i \leq n$.

Case 3.1: $1 \leq i \leq n-1$ : 


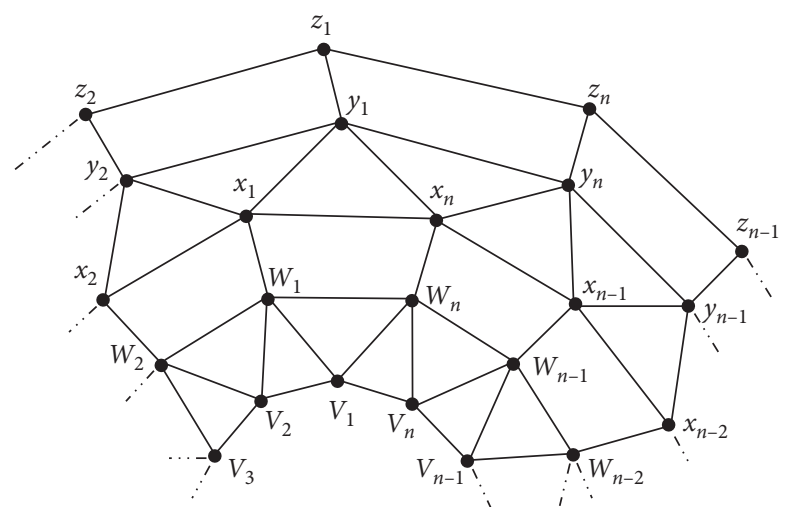

Figure 3: The graph of $n$-dimensional convex polytope $K_{n}$.

$$
\begin{aligned}
P_{H}\left(u^{\prime}, v^{\prime}\right): u^{\prime}= & \left\{z_{j}: 1 \leq j \leq n\right\} \sim\left\{y_{n-j}: 0 \leq j \leq n-1\right\} \sim\left\{x_{n-j}: 0 \leq j \leq n-i-1\right\} \sim\left\{w_{j}: i+1 \leq j \leq n\right\} \sim v_{1} \\
& \sim\left\{v_{n-j}: 0 \leq j \leq n-i-1\right\} \sim\left\{w_{i-j} v_{i-j}: 0 \leq j \leq i-2\right\} \sim w_{1} \sim\left\{x_{j}: 1 \leq j \leq i\right\}=v^{\prime} .
\end{aligned}
$$

Case 3.2: $i=n$ :

$$
\begin{aligned}
P_{H}\left(u^{\prime}, v^{\prime}\right): u^{\prime}= & \left\{z_{j}: 1 \leq j \leq n\right\} \sim\left\{y_{n-j}: 0 \leq j \leq n-1\right\} \sim\left\{x_{j}: 1 \leq j \leq n-1\right\} \sim\left\{w_{n-j}: 1 \leq j \leq n-1\right\} \\
& \sim\left\{v_{j}: 1 \leq j \leq n\right\} \sim w_{n} x_{n}=v^{\prime} .
\end{aligned}
$$

Case 4: $u^{\prime}=z_{1}$ and $v^{\prime}=w_{i}, 1 \leq i \leq n$.

Case 4.1: $1 \leq i \leq n-1$ :

$$
\begin{aligned}
P_{H}\left(u^{\prime}, v^{\prime}\right): u^{\prime}= & \left\{z_{j}: 1 \leq j \leq n\right\} \sim\left\{y_{n-j}: 0 \leq j \leq n-1\right\} \sim\left\{x_{j}: 1 \leq j \leq n\right\} \sim\left\{w_{n-j}: 0 \leq j \leq n-i-1\right\} \sim\left\{v_{j}: i+1 \leq j \leq n\right\} \\
& \sim\left\{v_{j} w_{j}: 1 \leq j \leq i\right\}=v^{\prime} .
\end{aligned}
$$

Case 4.2: $i=n$ :

$$
\begin{aligned}
P_{H}\left(u^{\prime}, v^{\prime}\right): u^{\prime}= & \left\{z_{j}: 1 \leq j \leq n\right\} \sim\left\{y_{n-j}: 0 \leq j \leq n-1\right\} \sim\left\{x_{j}: 1 \leq j \leq n-2\right\} \sim\left\{w_{n-j}: 2 \leq j \leq n-1\right\} \sim\left\{v_{j}: 1 \leq j \leq n\right\} \\
& \sim w_{n-1} x_{n-1} x_{n} w_{n}=v^{\prime} .
\end{aligned}
$$

Case 5: $u^{\prime}=z_{1}$ and $v^{\prime}=v_{i}, 1 \leq i \leq n$.

Case 5.1: $1 \leq i \leq n-1$ :

$$
\begin{aligned}
P_{H}\left(u^{\prime}, v^{\prime}\right): u^{\prime}= & \left\{z_{j}: 1 \leq j \leq n\right\} \sim\left\{y_{n-j}: 0 \leq j \leq n-1\right\} \sim\left\{x_{j}: 1 \leq j \leq n\right\} \sim\left\{w_{n-j}: 0 \leq j \leq n-i\right\} \sim\left\{v_{j}: i+1 \leq j \leq n\right\} \\
& \sim\left\{v_{j} w_{j}: 1 \leq j \leq i-1\right\} \sim v_{i}=v^{\prime} .
\end{aligned}
$$


Case 5.2: $i=n$ :

$$
P_{H}\left(u^{\prime}, v^{\prime}\right): u^{\prime}=\left\{z_{j}: 1 \leq j \leq n\right\} \sim\left\{y_{n-j}: 0 \leq j \leq n-1\right\} \sim\left\{x_{j}: 1 \leq j \leq n\right\} \sim\left\{w_{n-j}: 0 \leq j \leq n-1\right\} \sim\left\{v_{j}: 1 \leq j \leq n\right\}=v^{\prime} .
$$

Case 6: $u^{\prime}=y_{1}$ and $v^{\prime}=z_{i}, 1 \leq i \leq n$ :

Case 6.1: $1 \leq i \leq n-1$ :

$$
\begin{aligned}
P_{H}\left(u^{\prime}, v^{\prime}\right): u^{\prime}= & \left\{y_{j} x_{j}: 1 \leq j \leq i\right\} \sim\left\{x_{j}: i+1 \leq j \leq n-1\right\} \sim\left\{w_{n-j}: 1 \leq j \leq n-1\right\} \sim\left\{v_{j}: 1 \leq j \leq n\right\} \\
& \sim\left\{y_{n-j}: 0 \leq j \leq n-i-1\right\} \sim\left\{z_{j}: i+1 \leq j \leq n\right\} \sim\left\{z_{j}: 1 \leq j \leq i\right\}=v^{\prime} .
\end{aligned}
$$

Case 6.2: $i=n$ :

$$
\begin{aligned}
P_{H}\left(u^{\prime}, v^{\prime}\right): u^{\prime}= & y_{1} \sim\left\{z_{j}: 1 \leq j \leq n-1\right\} \sim\left\{y_{n-j}: 1 \leq j \leq n-2\right\} \sim\left\{x_{j}: 1 \leq j \leq n-1\right\} \sim\left\{w_{n-j}: 1 \leq j \leq n-1\right\} \\
& \sim\left\{v_{j}: 1 \leq j \leq n\right\} \sim w_{n} x_{n} y_{n} z_{n}=v^{\prime} .
\end{aligned}
$$

Case 7: $u^{\prime}=y_{1}$ and $v^{\prime}=y_{i}, 2 \leq i \leq n$

Case 7.1: $2 \leq i \leq n-1$ :

$$
\begin{aligned}
P_{H}\left(u^{\prime}, v^{\prime}\right): u^{\prime}= & y_{1} \sim\left\{z_{j}: 1 \leq j \leq n\right\} \sim\left\{y_{n-j}: 0 \leq j \leq n-i-1\right\} \sim\left\{x_{j}: i \leq j \leq n\right\} \sim\left\{w_{n-j}: 0 \leq j \leq n-2\right\} \\
& \sim\left\{v_{j}: 2 \leq j \leq n\right\} \sim v_{1} w_{1} \sim\left\{x_{j-1} y_{j}: 2 \leq j \leq i\right\}=v^{\prime} .
\end{aligned}
$$

Case 7.2: $i=n$ :

$$
\begin{aligned}
P_{H}\left(u^{\prime}, v^{\prime}\right): u^{\prime}= & y_{1} z_{1} \sim\left\{z_{n-j}: 0 \leq j \leq n-2\right\} \sim\left\{y_{j}: 2 \leq j \leq n-1\right\} \sim\left\{x_{n-j}: 1 \leq j \leq n-1\right\} \sim\left\{w_{j}: 1 \leq j \leq n-1\right\} \\
& \sim\left\{v_{n-j}: 0 \leq j \leq n-1\right\} \sim w_{n} x_{n} y_{n}=v^{\prime} .
\end{aligned}
$$

Case 8: $u^{\prime}=y_{1}$ and $v^{\prime}=x_{i}, 1 \leq i \leq n$.

Case 8.1: $1 \leq i \leq n-1$ :

$$
\begin{aligned}
P_{H}\left(u^{\prime}, v^{\prime}\right): u^{\prime}= & y_{1} z_{1} \sim\left\{z_{n-j}: 0 \leq j \leq n-2\right\} \sim\left\{y_{j}: 2 \leq j \leq n\right\} \sim\left\{x_{n-j}: 0 \leq j \leq n-i-1\right\} \sim w_{i+1} v_{i+1} \\
& \sim\left\{v_{j} w_{j}: i+2 \leq j \leq n\right\} \sim\left\{v_{j}: 1 \leq j \leq i\right\} \sim\left\{w_{i-j}: 0 \leq j \leq i-1\right\}\left\{x_{j}: 1 \leq j \leq i\right\}=v^{\prime} .
\end{aligned}
$$

Case 8.2: $i=n$ : 


$$
\begin{aligned}
P_{H}\left(u^{\prime}, v^{\prime}\right): u^{\prime}= & y_{1} \sim\left\{z_{j}: 1 \leq j \leq n\right\} \sim\left\{y_{n-j}: 0 \leq j \leq n-2\right\} \sim\left\{x_{j}: 1 \leq j \leq n-1\right\} \sim\left\{w_{n-j}: 1 \leq j \leq n-1\right\} \\
& \sim\left\{v_{j}: 1 \leq j \leq n\right\} \sim w_{n} x_{n}=v^{\prime} .
\end{aligned}
$$

Case 9: $u^{\prime}=y_{1}$ and $v^{\prime}=w_{i}, 1 \leq i \leq n$.

Case 9.1: $1 \leq i \leq n-1$ :

$$
\begin{aligned}
P_{H}\left(u^{\prime}, v^{\prime}\right): u^{\prime}= & y_{1} \sim\left\{z_{j}: 1 \leq j \leq n\right\} \sim\left\{y_{n-j}: 0 \leq j \leq n-2\right\} \sim\left\{x_{j}: 1 \leq j \leq n\right\} \sim\left\{w_{n-j}: 0 \leq j \leq n-i-1\right\} \\
& \sim\left\{v_{j}: i+1 \leq j \leq n\right\} \sim\left\{v_{j} w_{j}: 1 \leq j \leq i\right\}=v^{\prime} .
\end{aligned}
$$

Case 9.2: $i=n$ :

$$
\begin{aligned}
P_{H}\left(u^{\prime}, v^{\prime}\right): u^{\prime}= & y_{1} z_{1} \sim\left\{z_{n-j}: 0 \leq j \leq n-2\right\} \sim\left\{y_{j}: 2 \leq j \leq n\right\} \sim\left\{x_{n-j}: 0 \leq j \leq n-1\right\} \sim\left\{w_{j}: 1 \leq j \leq n-1\right\} \\
& \sim\left\{v_{n-j}: 0 \leq j \leq n-1\right\} \sim w_{n}=v^{\prime} .
\end{aligned}
$$

Case 10: $u^{\prime}=y_{1}$ and $v^{\prime}=v_{i}, 1 \leq i \leq n$.

Case 10.1: $1 \leq i \leq n-1$ :

$$
\begin{aligned}
P_{H}\left(u^{\prime}, v^{\prime}\right): u^{\prime}= & y_{1} \sim\left\{z_{j}: 1 \leq j \leq n\right\} \sim\left\{y_{n-j}: 0 \leq j \leq n-2\right\} \sim\left\{x_{j}: 1 \leq j \leq n\right\} \sim\left\{w_{n-j}: 0 \leq j \leq n-i\right\} \\
& \sim\left\{v_{j}: i+1 \leq j \leq n\right\} \sim\left\{v_{j} w_{j}: 1 \leq j \leq i-1\right\} \sim v_{i}=v^{\prime} .
\end{aligned}
$$

Case 10.2: $i=n$ :

$$
\begin{aligned}
P_{H}\left(u^{\prime}, v^{\prime}\right): u^{\prime}= & y_{1} \sim\left\{z_{j}: 1 \leq j \leq n\right\} \sim\left\{y_{n-j}: 0 \leq j \leq n-2\right\} \sim\left\{x_{j}: 1 \leq j \leq n\right\} \sim\left\{w_{n-j}: 0 \leq j \leq n-1\right\} \\
& \sim\left\{v_{j}: 1 \leq j \leq n\right\}=v^{\prime} .
\end{aligned}
$$

Case 11: $u^{\prime}=x_{1}$ and $v^{\prime}=z_{i}, 1 \leq i \leq n$.

Case 11.1: $1 \leq i \leq n-1$ :

$$
\begin{aligned}
P_{H}\left(u^{\prime}, v^{\prime}\right): u^{\prime}= & x_{1} \sim\left\{y_{j} x_{j}: 2 \leq j \leq i\right\} \sim\left\{w_{i-j}: 0 \leq j \leq i-1\right\} \sim\left\{w_{n-j}: 0 \leq j \leq n-i-2\right\} \sim\left\{v_{j}: i+2 \leq j \leq n\right\} \\
& \sim\left\{v_{j}: 1 \leq j \leq i+1\right\} \sim w_{i+1} \sim\left\{x_{j}: i+1 \leq j \leq n\right\} \sim y_{1} \sim\left\{y_{n-j}: 0 \leq j \leq n-i-1\right\} \sim\left\{z_{j}: i+1 \leq j \leq n\right\} \\
& \sim\left\{z_{j}: 1 \leq j \leq i\right\}=v^{\prime} .
\end{aligned}
$$

Case 11.2: $i=n$ : 


$$
\begin{aligned}
P_{H}\left(u^{\prime}, v^{\prime}\right): u^{\prime}= & x_{1} w_{1} \sim\left\{v_{j}: 1 \leq j \leq n\right\} \sim\left\{w_{n-j}: 0 \leq j \leq n-2\right\} \sim\left\{x_{j}: 2 \leq j \leq n\right\} \sim\left\{y_{n-j}: 0 \leq j \leq n-1\right\} \\
& \sim\left\{z_{j}: 1 \leq j \leq n\right\}=v^{\prime} .
\end{aligned}
$$

Case 12: $u^{\prime}=x_{1}$ and $v^{\prime}=y_{i}, 1 \leq i \leq n$.

Case 12.1: $i=1$ :

$$
\begin{aligned}
P_{H}\left(u^{\prime}, v^{\prime}\right): u^{\prime}= & x_{1} w_{1} v_{1} \sim\left\{v_{n-j}: 0 \leq j \leq n-2\right\} \sim\left\{w_{j}: 2 \leq j \leq n\right\} \sim\left\{x_{n-j}: 0 \leq j \leq n-2\right\} \sim\left\{y_{j}: 2 \leq j \leq n\right\} \\
& \sim\left\{z_{n-j}: 0 \leq j \leq n-1\right\} \sim y_{1}=v^{\prime} .
\end{aligned}
$$

Case 12.2: $2 \leq i \leq n-1$ :

$$
\begin{gathered}
P_{H}\left(u^{\prime}, v^{\prime}\right): u^{\prime}=x_{1} w_{1} v_{1} \sim\left\{v_{n-j}: 0 \leq j \leq n-2\right\} \sim\left\{w_{j}: 2 \leq j \leq n\right\} \sim\left\{x_{n-j}: 0 \leq j \leq n-i\right\} \sim\left\{y_{j}: i+1 \leq j \leq n\right\} \\
\sim\left\{z_{n-j}: 0 \leq j \leq n-1\right\} \sim y_{1} \sim\left\{y_{j} x_{j}: 2 \leq j \leq i-1\right\} \sim y_{i}=v^{\prime} .
\end{gathered}
$$

Case 12.3: $i=n$ :

$$
\begin{aligned}
P_{H}\left(u^{\prime}, v^{\prime}\right): u^{\prime}= & x_{1} w_{1} v_{1} \sim\left\{z_{n-j}: 0 \leq j \leq n-2\right\} \sim\left\{y_{j}: 2 \leq j \leq n-1\right\} \sim\left\{x_{n-j}: 1 \leq j \leq n-2\right\} \sim\left\{w_{j}: 2 \leq j \leq n-1\right\} \\
& \sim\left\{v_{n-j}: 0 \leq j \leq n-2\right\} \sim w_{1} v_{1} w_{n} x_{n} y_{n}=v^{\prime}
\end{aligned}
$$

Case 13: $u^{\prime}=x_{1}$ and $v^{\prime}=x_{i}, 2 \leq i \leq n$.

Case 13.1: $i=2$ :

$$
\begin{aligned}
P_{H}\left(u^{\prime}, v^{\prime}\right): u^{\prime}= & x_{1} \sim\left\{y_{j}: 1 \leq j \leq n-1\right\} \sim\left\{z_{n-j}: 1 \leq j \leq n-1\right\} \sim z_{n} y_{n} \sim\left\{x_{n-j}: 0 \leq j \leq n-3\right\} \sim w_{3} v_{3} \sim\left\{v_{j} w_{j}: 4 \leq j \leq n\right\} \\
& \sim v_{1} w_{1} v_{2} w_{2} x_{2}=v^{\prime} .
\end{aligned}
$$

Case 13.2: $3 \leq i \leq n-1$ :

$$
\begin{aligned}
P_{H}\left(u^{\prime}, v^{\prime}\right): u^{\prime}= & x_{1} y_{1} \sim\left\{y_{j} x_{j}: 2 \leq j \leq i-1\right\} \sim\left\{y_{j}: i \leq j \leq n-1\right\} \sim\left\{z_{n-j}: 1 \leq j \leq n-1\right\} \sim z_{n} y_{n} \\
& \sim\left\{x_{n-j}: 0 \leq j \leq n-i-1\right\} \sim w_{i+1} v_{i+1} \sim\left\{v_{j} w_{j}: i+2 \leq j \leq n\right\} \sim\left\{v_{j} w_{j}: 1 \leq j \leq i\right\} \sim x_{i}=v^{\prime} .
\end{aligned}
$$

Case 13.3: $i=n$ :

$$
\begin{aligned}
P_{H}\left(u^{\prime}, v^{\prime}\right): u^{\prime}= & x_{1} w_{1} \sim\left\{v_{j}: 1 \leq j \leq n\right\} \sim\left\{w_{n-j}: 0 \leq j \leq n-2\right\} \sim\left\{x_{j}: 2 \leq j \leq n-1\right\} \sim\left\{y_{n-j}: 1 \leq j \leq n-1\right\} \\
& \sim\left\{z_{j}: 1 \leq j \leq n\right\} \sim y_{n} x_{n}=v^{\prime} .
\end{aligned}
$$

Case 14: $u^{\prime}=x_{1}$ and $v^{\prime}=w_{i}, 1 \leq i \leq n$.

Case 14.1: $1 \leq i \leq n-1$ : 


$$
\begin{aligned}
P_{H}\left(u^{\prime}, v^{\prime}\right): u^{\prime}= & x_{1} y_{1} \sim\left\{z_{j}: 1 \leq j \leq n\right\} \sim\left\{y_{n-j}: 0 \leq j \leq n-2\right\} \sim\left\{x_{j}: 2 \leq j \leq n\right\} \sim\left\{w_{n-j}: 0 \leq j \leq n-i-1\right\} \\
& \sim\left\{v_{j}: i+1 \leq j \leq n\right\} \sim\left\{v_{j} w_{j}: 1 \leq j \leq i\right\}=v^{\prime} .
\end{aligned}
$$

Case 14.2: $i=n$ :

$$
\begin{aligned}
P_{H}\left(u^{\prime}, v^{\prime}\right): u^{\prime}= & x_{1} w_{1} \sim\left\{v_{j}: 1 \leq j \leq n\right\} \sim\left\{w_{n-j}: 1 \leq j \leq n-2\right\} \sim\left\{x_{j}: 2 \leq j \leq n-1\right\} \sim\left\{y_{n-j}: 1 \leq j \leq n-1\right\} \\
& \sim\left\{z_{j}: 1 \leq j \leq n\right\} \sim y_{n} x_{n} w_{n}=v^{\prime} .
\end{aligned}
$$

Case 15: $u^{\prime}=x_{1}$ and $v^{\prime}=v_{i}, 1 \leq i \leq n$.

Case 15.1: $1 \leq i \leq n-1$ :

$$
\begin{aligned}
P_{H}\left(u^{\prime}, v^{\prime}\right): u^{\prime}= & x_{1} y_{1} \sim\left\{z_{j}: 1 \leq j \leq n\right\} \sim\left\{y_{n-j}: 0 \leq j \leq n-2\right\} \sim\left\{x_{j}: 2 \leq j \leq n\right\} \sim\left\{w_{n-j}: 0 \leq j \leq n-i\right\} \\
& \sim\left\{v_{j}: i+1 \leq j \leq n\right\} \sim\left\{v_{j} w_{j}: 1 \leq j \leq i-1\right\} \sim v_{i}=v^{\prime} .
\end{aligned}
$$

Case 15.2: $i=n$ :

$$
\begin{aligned}
P_{H}\left(u^{\prime}, v^{\prime}\right): u^{\prime}= & x_{1} y_{1} \sim\left\{z_{j}: 1 \leq j \leq n\right\} \sim\left\{y_{n-j}: 0 \leq j \leq n-2\right\} \sim\left\{x_{j}: 2 \leq j \leq n\right\} \\
& \sim\left\{w_{n-j}: 0 \leq j \leq n-1\right\} \sim\left\{v_{j}: 1 \leq j \leq n\right\}=v^{\prime} .
\end{aligned}
$$

Case 16: $u^{\prime}=w_{1}$ and $v^{\prime}=z_{i}, 1 \leq i \leq n$.

Case 16.1: $1 \leq i \leq n-1$ :

$$
\begin{aligned}
P_{H}\left(u^{\prime}, v^{\prime}\right): u^{\prime}= & w_{1} v_{1} \sim\left\{v_{n-j}: 0 \leq j \leq n-2\right\} \sim\left\{w_{j}: 2 \leq j \leq n\right\} \sim x_{n} \sim\left\{x_{j}: 1 \leq j \leq i\right\} \sim\left\{y_{i-j}: 0 \leq j \leq i-1\right\} \\
& \sim\left\{y_{n-j}: 0 \leq j \leq n-i-1\right\} \sim\left\{z_{j}: i+1 \leq j \leq n\right\} \sim\left\{z_{j}: 1 \leq j \leq i\right\}=v^{\prime} .
\end{aligned}
$$

Case 16.2: $i=n$ :

$$
\begin{aligned}
P_{H}\left(u^{\prime}, v^{\prime}\right): u^{\prime}= & w_{1} \sim\left\{v_{j}: 1 \leq j \leq n\right\} \sim\left\{w_{n-j}: 0 \leq j \leq n-2\right\} \sim\left\{x_{j}: 2 \leq j \leq n\right\} \sim\left\{y_{n-j}: 0 \leq j \leq n-2\right\} \\
& \sim x_{1} y_{1} \sim\left\{z_{j}: 1 \leq j \leq n\right\}=v^{\prime} .
\end{aligned}
$$

Case 17: $u^{\prime}=w_{1}$ and $v^{\prime}=y_{i}, 1 \leq i \leq n$.

Case 17.1: $1 \leq i \leq n-1$ : 


$$
\begin{gathered}
P_{H}\left(u^{\prime}, v^{\prime}\right): u^{\prime}=w_{1} w_{n} v_{1} \sim\left\{v_{n-j}: 0 \leq j \leq n-2\right\} \sim\left\{w_{j}: 2 \leq j \leq n-1\right\} \sim\left\{x_{n-j} y_{n-j}: 1 \leq j \leq n-i-1\right\} \\
\sim\left\{x_{i-j}: 0 \leq j \leq i-1\right\} \sim x_{n} y_{n} \sim\left\{z_{n-j}: 0 \leq j \leq n-1\right\} \sim\left\{y_{j}: 1 \leq j \leq i\right\}=v^{\prime} .
\end{gathered}
$$

Case 17.2: $i=n$ :

$$
\begin{aligned}
P_{H}\left(u^{\prime}, v^{\prime}\right): u^{\prime}= & w_{1} v_{1} \sim\left\{v_{n-j}: 0 \leq j \leq n-2\right\} \sim\left\{w_{j}: 2 \leq j \leq n\right\} \sim\left\{x_{n-j}: 0 \leq j \leq n-1\right\} \sim\left\{y_{j}: 1 \leq j \leq n-1\right\} \\
& \sim\left\{z_{n-j}: 1 \leq j \leq n-1\right\} \sim z_{n} y_{n}=v^{\prime} .
\end{aligned}
$$

Case 18: $u^{\prime}=w_{1}$ and $v^{\prime}=x_{i}, 1 \leq i \leq n$.

Case 18.1: $1 \leq i \leq n-1$ :

$$
\begin{aligned}
P_{H}\left(u^{\prime}, v^{\prime}\right): u^{\prime}= & w_{1} v_{1} \sim\left\{v_{n-j}: 0 \leq j \leq n-2\right\} \sim\left\{w_{j}: 2 \leq j \leq n\right\} \sim\left\{x_{n-j}: 0 \leq j \leq n-i-1\right\} \sim\left\{y_{j}: i+1 \leq j \leq n\right\} \\
& \sim\left\{z_{n-j}: 0 \leq j \leq n-1\right\} \sim\left\{y_{j} x_{j}: 1 \leq j \leq i\right\}=v^{\prime} .
\end{aligned}
$$

Case 18.2: $i=n$ :

$$
\begin{aligned}
P_{H}\left(u^{\prime}, v^{\prime}\right): u^{\prime}= & w_{1} \sim\left\{v_{j}: 1 \leq j \leq n\right\} \sim\left\{w_{n-j}: 0 \leq j \leq n-2\right\} \sim\left\{x_{j}: 2 \leq j \leq n-1\right\} \sim\left\{y_{n-j}: 1 \leq j \leq n-2\right\} \\
& \sim x_{1} y_{1} \sim\left\{z_{j}: 1 \leq j \leq n\right\} \sim y_{n} x_{n}=v^{\prime}
\end{aligned}
$$

Case 19: $u^{\prime}=w_{1}$ and $v^{\prime}=w_{i}, 2 \leq i \leq n$.

Case 19.1: $2 \leq i \leq n-1$ :

$$
\begin{aligned}
P_{H}\left(u^{\prime}, v^{\prime}\right): u^{\prime}= & w_{1} x_{1} y_{1} \sim\left\{z_{j}: 1 \leq j \leq n\right\} \sim\left\{y_{n-j}: 0 \leq j \leq n-2\right\} \sim\left\{x_{j}: 2 \leq j \leq n\right\} \sim\left\{w_{n-j}: 0 \leq j \leq n-i-1\right\} \\
& \sim\left\{v_{j}: i+1 \leq j \leq n\right\} \sim v_{1} \sim\left\{v_{j} w_{j}: 2 \leq j \leq i\right\}=v^{\prime} .
\end{aligned}
$$

Case 19.2: $i=n$ :

$$
\begin{gathered}
P_{H}\left(u^{\prime}, v^{\prime}\right): u^{\prime}=w_{1} x_{1} y_{1} z_{1} \sim\left\{z_{n-j}: 0 \leq j \leq n-2\right\} \sim\left\{y_{j}: 2 \leq j \leq n\right\} \sim\left\{x_{n-j}: 0 \leq j \leq n-2\right\} \\
\sim\left\{w_{j}: 2 \leq j \leq n-1\right\} \sim\left\{v_{n-j}: 1 \leq j \leq n-1\right\} \sim v_{n} w_{n}=v^{\prime} .
\end{gathered}
$$

Case 20: $u^{\prime}=w_{1}$ and $v^{\prime}=v_{i}, 1 \leq i \leq n$.

Case 20.1: $i=1$ : 


$$
\begin{aligned}
P_{H}\left(u^{\prime}, v^{\prime}\right): u^{\prime}= & w_{1} x_{1} y_{1} \sim\left\{z_{j}: 1 \leq j \leq n\right\} \sim\left\{y_{n-j}: 0 \leq j \leq n-2\right\} \sim\left\{x_{j}: 2 \leq j \leq n\right\} \sim\left\{w_{n-j}: 0 \leq j \leq n-2\right\} \\
& \sim\left\{v_{j}: 2 \leq j \leq n\right\} \sim v_{1}=v^{\prime} .
\end{aligned}
$$

Case 20.2: $2 \leq i \leq n-1$ :

$$
\begin{aligned}
P_{H}\left(u^{\prime}, v^{\prime}\right): u^{\prime}= & w_{1} x_{1} y_{1} \sim\left\{z_{j}: 1 \leq j \leq n\right\} \sim\left\{y_{n-j}: 0 \leq j \leq n-2\right\} \sim\left\{x_{j}: 2 \leq j \leq n\right\} \sim\left\{w_{n-j}: 0 \leq j \leq n-i\right\} \\
& \sim\left\{v_{j}: i+1 \leq j \leq n\right\} \sim v_{1} \sim\left\{v_{j} w_{j}: 2 \leq j \leq i-1\right\} \sim v_{i}=v^{\prime} .
\end{aligned}
$$

Case 20.3: $i=n$ :

$$
\begin{aligned}
P_{H}\left(u^{\prime}, v^{\prime}\right): u^{\prime}= & w_{1} x_{1} y_{1} z_{1} \sim\left\{z_{n-j}: 0 \leq j \leq n-2\right\} \sim\left\{y_{j}: 2 \leq j \leq n\right\} \sim\left\{x_{n-j}: 0 \leq j \leq n-2\right\} \sim\left\{w_{j}: 2 \leq j \leq n\right\} \\
& \sim\left\{v_{j}: 1 \leq j \leq n\right\}=v^{\prime} .
\end{aligned}
$$

Case 21: $u^{\prime}=v_{1}$ and $v^{\prime}=z_{i}, 1 \leq i \leq n$.

Case 21.1: $1 \leq i \leq n-1$ :

$$
\begin{aligned}
P_{H}\left(u^{\prime}, v^{\prime}\right): u^{\prime}= & v_{1} \sim\left\{v_{n-j}: 0 \leq j \leq n-2\right\} \sim\left\{w_{j}: 1 \leq j \leq n\right\} \sim\left\{x_{n-j}: 0 \leq j \leq n-i-1\right\} \sim\left\{y_{j}: i+2 \leq j \leq n\right\} \\
& \sim\left\{y_{j} x_{j}: 1 \leq j \leq i\right\} \sim y_{i+1} \sim\left\{z_{j}: i+1 \leq j \leq n\right\} \sim\left\{z_{j}: 1 \leq j \leq i\right\}=v^{\prime} .
\end{aligned}
$$

Case 21.2: $i=n$ :

$P_{H}\left(u^{\prime}, v^{\prime}\right): u^{\prime}=\left\{v_{j}: 1 \leq j \leq n\right\} \sim\left\{w_{n-j}: 0 \leq j \leq n-1\right\} \sim\left\{x_{j}: 1 \leq j \leq n\right\} \sim\left\{y_{n-j}: 0 \leq j \leq n-1\right\} \sim\left\{z_{j}: 1 \leq j \leq n\right\}=v^{\prime}$.

Case 22: $u^{\prime}=v_{1}$ and $v^{\prime}=y_{i}, 1 \leq i \leq n$.

Case 22.1: $1 \leq i \leq n-1$ :

$$
\begin{aligned}
P_{H}\left(u^{\prime}, v^{\prime}\right): u^{\prime}= & v_{1} \sim\left\{v_{n-j}: 0 \leq j \leq n-2\right\} \sim\left\{w_{j}: 1 \leq j \leq n\right\} \sim\left\{x_{n-j}: 0 \leq j \leq n-i\right\} \sim\left\{y_{j}: i+1 \leq j \leq n\right\} \\
& \sim\left\{z_{n-j}: 0 \leq j \leq n-1\right\} \sim\left\{y_{j} x_{j}: 1 \leq j \leq i-1\right\} \sim y_{i}=v^{\prime} .
\end{aligned}
$$

Case 22.2: $i=n$ :

$$
\begin{aligned}
P_{H}\left(u^{\prime}, v^{\prime}\right): u^{\prime}= & v_{1} \sim\left\{v_{n-j}: 0 \leq j \leq n-2\right\} \sim\left\{w_{j}: 1 \leq j \leq n\right\} \sim\left\{x_{n-j}: 0 \leq j \leq n-1\right\} \sim\left\{y_{j}: 1 \leq j \leq n-1\right\} \\
& \sim\left\{z_{n-j}: 1 \leq j \leq n-1\right\} \sim z_{n} y_{n}=v^{\prime} .
\end{aligned}
$$

Case 23: $u^{\prime}=v_{1}$ and $v^{\prime}=x_{i}, 1 \leq i \leq n$.

Case 23.1: $1 \leq i \leq n-1$ : 


$$
\begin{aligned}
P_{H}\left(u^{\prime}, v^{\prime}\right): u^{\prime}= & v_{1} \sim\left\{v_{n-j}: 0 \leq j \leq n-2\right\} \sim\left\{w_{j}: 1 \leq j \leq n\right\} \\
& \sim\left\{x_{n-j}: 0 \leq j \leq n-i-1\right\} \sim\left\{y_{j}: i+1 \leq j \leq n\right\} \sim\left\{z_{n-j}: 0 \leq j \leq n-1\right\} \sim\left\{y_{j} x_{j}: 1 \leq j \leq i\right\}=v^{\prime}
\end{aligned}
$$

Case 23.2: $i=n$ :

$$
\begin{aligned}
P_{H}\left(u^{\prime}, v^{\prime}\right): u^{\prime}= & \left\{v_{j}: 1 \leq j \leq n\right\} \sim\left\{w_{n-j}: 0 \leq j \leq n-1\right\} \sim\left\{x_{j}: 1 \leq j \leq n-1\right\} \sim\left\{y_{n-j}: 1 \leq j \leq n-1\right\} \\
& \sim\left\{z_{j}: 1 \leq j \leq n\right\} \sim y_{n} x_{n}=v^{\prime} .
\end{aligned}
$$

Case 24: $u^{\prime}=v_{1}$ and $v^{\prime}=w_{i}, 1 \leq i \leq n$.

Case 24.1: $1 \leq i \leq n-1$ :

$$
\begin{aligned}
P_{H}\left(u^{\prime}, v^{\prime}\right): u^{\prime}= & \left\{v_{j} w_{j}: 1 \leq j \leq i-1\right\} \sim\left\{v_{j}: i \leq j \leq n\right\} \sim\left\{w_{n-j}: 0 \leq j \leq n-i-1\right\} \sim\left\{x_{j}: i+1 \leq j \leq n\right\} \\
& \sim\left\{y_{n-j}: 0 \leq j \leq n-2\right\} \sim\left\{z_{j}: 2 \leq j \leq n\right\} \sim z_{1} y_{1} \sim\left\{x_{j}: 1 \leq j \leq i\right\} \sim w_{i}=v^{\prime} .
\end{aligned}
$$

Case 24.2: $i=n$ :

$$
\begin{aligned}
P_{H}\left(u^{\prime}, v^{\prime}\right): u^{\prime}= & v_{1} \sim\left\{v_{n-j}: 0 \leq j \leq n-2\right\} \sim\left\{w_{j}: 1 \leq j \leq n-1\right\} \sim\left\{x_{n-j}: 1 \leq j \leq n-1\right\} \sim\left\{y_{j}: 1 \leq j \leq n-1\right\} \\
& \sim\left\{z_{n-j}: 1 \leq j \leq n-1\right\} \sim z_{n} y_{n} x_{n} w_{n}=v^{\prime}
\end{aligned}
$$

Case 25: $u^{\prime}=v_{1}$ and $v^{\prime}=v_{i}, 2 \leq i \leq n$.

Case 25.1: $2 \leq i \leq n-1$ :

$$
\begin{aligned}
P_{H}\left(u^{\prime}, v^{\prime}\right): u^{\prime}= & v_{1} \sim\left\{v_{n-j}: 0 \leq j \leq n-i-1\right\} \sim\left\{w_{j}: i \leq j \leq n\right\} \sim\left\{x_{n-j}: 0 \leq j \leq n-2\right\} \sim\left\{y_{j}: 2 \leq j \leq n\right\} \\
& \sim\left\{z_{n-j}: 0 \leq j \leq n-1\right\} \sim y_{1} x_{1} \sim\left\{w_{j-1} v_{j}: 2 \leq j \leq i\right\}=v^{\prime}
\end{aligned}
$$

Case 25.2: $i=n$ :

$$
\begin{aligned}
P_{H}\left(u^{\prime}, v^{\prime}\right): u^{\prime}= & v_{1} w_{1} x_{1} y_{1} \sim\left\{z_{j}: 1 \leq j \leq n\right\} \sim\left\{y_{n-j}: 0 \leq j \leq n-2\right\} \sim\left\{x_{j}: 2 \leq j \leq n\right\} \sim\left\{w_{n-j}: 0 \leq j \leq n-2\right\} \\
& \sim\left\{v_{j}: 2 \leq j \leq n\right\}=v^{\prime} .
\end{aligned}
$$

Existence of Hamiltonian paths between any two vertices of the $n$-dimensional convex polytope $K_{n}$ completes the proof. $\square$

Using Theorems 1 and 4, the following proposition computes the detour index of $K_{n}$.
Proposition 3. Let $G=K_{n}$, where $n \geq 3$. Then the detour index of $G$ is

$$
\omega(G)=\frac{5 n(5 n-1)^{2}}{2} .
$$




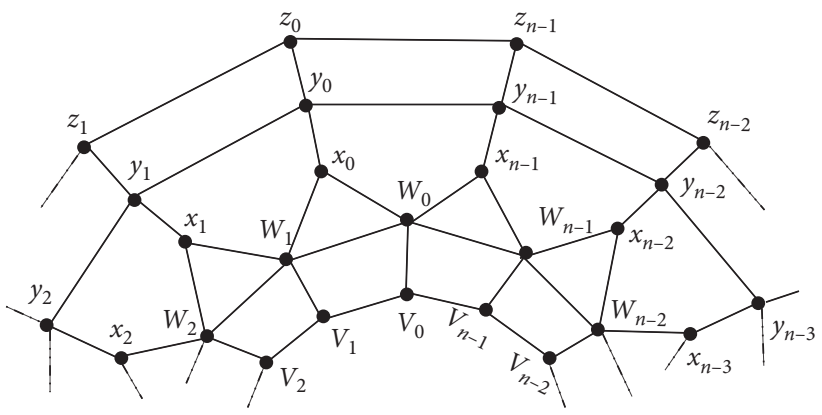

Figure 4: The graph of convex polytope $B_{n}$.

Proof. The number of vertices in graph $G$ is $5 n$. Replacing $5 n$ with $n$ in Theorem 1 completes the proof.

\section{A Family of Non-Hamilton-connected Convex Polytopes}

For $n \geq 4$, by $B_{n}$, we denote the graph of convex polytope defined in [24] which consists of $n$ number of 3-sided faces,
$2 n$ number of 4 -sided faces, and $n$ number of pentagonal faces, see Figure 4.

Mathematically, the vertex set of $B_{n}$ consists of four layers of vertices, i.e., $v_{i}, w_{i}, x_{i}, y_{i}$, and $z_{i}$. That is to say that $V\left(B_{n}\right)=\left\{v_{i}, w_{i}, x_{i}, y_{i}, z_{i}: 0 \leq i \leq n-1\right\}$. Accordingly, the edge set of $B_{n}$ is as follows:

$$
E\left(B_{n}\right)=\left\{v_{i} v_{i+1}, v_{i} w_{i}, w_{i} w_{i+1}, w_{i} x_{i}, w_{i+1} x_{i}, x_{i} y_{i}, y_{i} y_{i+1}, y_{i} z_{i}, z_{i} z_{i+1}: 0 \leq i \leq n-1\right\} .
$$

Note that arithmetics in the subscripts is performed modulo $n-1$. Layer comprising vertices $v_{i}(0 \leq i \leq n-1)$ is called the first layer. Similarly, layers composed by vertices $w_{i}(0 \leq i \leq n-1), \quad x_{i}(0 \leq i \leq n-1), \quad y_{i}(0 \leq i \leq n-1), \quad$ and $z_{i}(0 \leq i \leq n-1)$ are called second layer, third layer, fourth layer, and fifth layer, respectively. Note that first, second, fourth, and fifth layers form cycles of length $n$.

The following result shows that the infinite family $B_{n}$ of convex polytopes is non-Hamilton-connected.

Theorem 5. The n-dimensional convex polytope $B_{n}$, with $n \geq 4$, is non-Hamilton-connected.

Proof. It is enough to show that there exist two vertices in the $n$-dimensional convex polytope $B_{n}$ such that no Hamiltonian path exists between them.

It is easy to see that there exists no Hamiltonian path between any two vertices at distance two on the outer layer, i.e., between $z_{i}$ and $z_{i+2}$ if $n$ is even and between consecutive vertices on the outer layer, i.e., $z_{i}$ and $z_{i+1}$ if $n$ is odd, where $0 \leq i \leq n-1$ such that subscripts are taken modulo $n-1$. This shows that the $n$-dimensional convex polytope $B_{n}$ is nonHamilton-connected.

\section{Conclusion}

Determining whether or not a graph is Hamilton-connected is NP-complete graph. Thus, it is natural to study the Hamilton-connectivity of infinite families of graphs. In this paper, we have studied Hamilton-connectivity of certain infinite families of convex polytopes. We construct three infinite families of convex polytopes which have been shown to be Hamilton-connected. Moreover, one infinite family is shown to be non-Hamilton-connected which shows that not all the convex polytopes are Hamilton-connected. As a byproduct, we compute the detour index of Hamilton-connected families of convex polytopes.

As future directions, we propose the following problems:

Problem 1. Is there any other way to show Hamiltonconnectivity of a given graph?

Problem 2. Baca [18] introduced a family $D_{n}$ of convex polytopes. Determine whether $D_{n}$ is Hamilton-connected.

Problem 3. Imran et al. [57] introduced the family $T_{n}$ of convex polytopes. Determine whether or not $T_{n}$ is Hamilton-connected.

\section{Data Availability}

No data were used to support the findings of the study.

\section{Conflicts of Interest}

The authors declare that they have no conflicts of interest regarding the publication of the article.

\section{Authors' Contributions}

S. H. and A. K. devised the methodology and acquired funding. S. H. and S. K. carried out the formal analysis and data curation. S. H. and J.-B. L. wrote the original draft, reviewed the writing, and edited the manuscript. S. H., A. K., 
and J.-B. L. proofread the manuscript before its final submission. S. H. and A. K. contributed equally to this work.

\section{Acknowledgments}

Sakander Hayat was grateful to Dr. Muhammad Imran for providing the registration details to Mayura software. Sakander Hayat was supported by the Higher Education Commission, Pakistan, under Grant no. 20-11682/NRPU/ RGM/R\&D/HEC/2020. J-B. Liu was supported by Anhui Provincial Natural Science Foundation under Grant 2008085J01 and Natural Science Fund of Education Department of Anhui province under Grant no. KJ2020A0478.

\section{References}

[1] O. Ore, "Hamilton-connected graphs," Journal de Mathématiques Pures et Appliquées, vol. 42, pp. 21-27, 1963.

[2] R. Frucht, "A canonical representation of trivalent Hamiltonian graphs," Journal of Graph Theory, vol. 1, no. 1, pp. $45-60,1976$.

[3] V. S. Gordon, Y. L. Orlovich, and F. Werner, "Hamiltonian properties of triangular grid graphs," Discrete Mathematics, vol. 308, no. 24, pp. 6166-6188, 2008.

[4] S. Qiang, Z. Qain, and A. Yahui, "The Hamiltonicity of generalized honeycomb torus networks," Information Processing Letters, vol. 115, no. 2, pp. 104-111, 2005.

[5] I. A. Stewart, "Sufficient conditions for Hamiltonicity in multiswapped networks," Journal of Parallel and Distributed Computing, vol. 101, pp. 17-26, 2017.

[6] B. Wei, "Hamiltonian paths and Hamiltonian connectivity in graphs," Discrete Mathematics, vol. 121, no. 1-3, pp. 223-228, 1993.

[7] X. Yang, D. J. Evans, H. Lai, and G. M. Megson, "Generalized honeycomb torus is Hamiltonian," Information Processing Letters, vol. 92, no. 1, pp. 31-37, 2004.

[8] G. Chartrand, A. M. Hobbs, H. A. Jung, S. F. Kapoor, and C. S. J. A. Nash-Williams, "The square of a block is Hamiltonian connected," Journal of Combinatorial Theory, Series B, vol. 16, no. 3, pp. 290-292, 1974.

[9] C. Thomassen, "Hamiltonian-connected tournaments," Journal of Combinatorial Theory, Series B, vol. 28, no. 2, pp. 142-163, 1980.

[10] J.-M. Chang, J.-S. Yang, Y.-L. Wang, and Y. Cheng, "Panconnectivity, fault-tolerant Hamiltonicity and Hamiltonianconnectivity in alternating group graphs," Networks, vol. 44, no. 4, pp. 302-310, 2004.

[11] Z. Kewen, H.-J. Lai, and J. Zhou, "Hamiltonian-connected graphs," Computers \& Mathematics with Applications, vol. 55, no. 12, pp. 2707-2714, 2008.

[12] Q. Zhou and L. Wang, "Some sufficient spectral conditions on Hamilton-connected and traceable graphs," Linear and Multilinear Algebra, vol. 65, no. 2, pp. 224-234, 2017.

[13] Q. Zhou, L. Wang, and Y. Lu, "Wiener index and Harary index on Hamilton-connected graphs with large minimum degree," Discrete Applied Mathematics, vol. 247, pp. 180-185, 2018.

[14] J. Wei, Z. You, and H.-J. Lai, "Spectral analogues of Erdős' theorem on Hamilton-connected graphs," Applied Mathematics and Computation, vol. 340, pp. 242-250, 2019.

[15] R. W. Hung, F. Keshavarz-Kohjerdi, C. B. Lin, and J. S. Chen, "The Hamiltonian connectivity of alphabet supergrid graphs,"
International Journal of Applied Mathematics, vol. 49, no. 1, pp. 1-10, 2019.

[16] Q. Zhou, L. Wang, and Y. Lu, "Signless Laplacian spectral conditions for Hamilton-connected graphs with large minimum degree," Linear Algebra and Its Applications, vol. 592, pp. $48-64,2020$.

[17] A. Shabbir, M. F. Nadeem, and T. Zamfirescu, "The property of Hamiltonian connectedness in Toeplitz graphs," Complexity, vol. 2020, Article ID 5608720, 6 pages, 2020.

[18] M. Bača, "Face anti-magic labelings of convex polytopes," Utilitas Mathematica, vol. 55, pp. 221-226, 1999.

[19] M. Baca, "Labelings of two classes of convex polytopes," Utilitas Mathematica, vol. 34, pp. 24-31, 1988.

[20] M. Bača, "On magic labellings of convex polytopes," Annals of Discrete Mathematics, vol. 51, pp. 13-16, 1992.

[21] M. Miller, M. Baca, and J. A. MacDougall, "Vertex-magic total labeling of generalized Petersen graphs and convex polytopes," Journal of Combinatorial Mathematics and Combinatorial Computing, vol. 59, pp. 89-99, 2006.

[22] M. Imran, A. Q. Baig, and A. Ahmad, "Families of plane graphs with constant metric dimension," Utilitas Mathematica, vol. 88, pp. 43-57, 2012.

[23] M. Imran, S. A. U. H. Bokhary, and A. Q. Baig, "On the metric dimension of rotationally-symmetric convex polytopes," Journal of Algebra Combinatorics Discrete Structures and Applications, vol. 3, pp. 45-59, 2015.

[24] M. Imran and H. M. A. Siddiqui, "Computing the metric dimension of convex polytopes generated by wheel related graphs," Acta Mathematica Hungarica, vol. 149, no. 1, pp. 10-30, 2016.

[25] M. A. Malik and M. Sarwar, "On the metric dimension of two families of convex polytopes," Afrika Matematika, vol. 27, no. 1-2, pp. 229-238, 2016.

[26] M. Imran, S. A. u. H. Bokhary, A. Ahmad, and A. Semaničováfeňovčíková, "On classes of regular graphs with constant metric dimension," Acta Mathematica Scientia, vol. 33, no. 1, pp. 187-206, 2013.

[27] J. Kratica, V. Kovačević-Vujčić, M. Čangalović, and M. Stojanović, "Minimal doubly resolving sets and the strong metric dimension of some convex polytopes," Applied Mathematics and Computation, vol. 218, no. 19, pp. 9790-9801, 2012.

[28] H. Raza, S. Hayat, and X.-F. Pan, "On the fault-tolerant metric dimension of convex polytopes," Applied Mathematics and Computation, vol. 339, pp. 172-185, 2018.

[29] H. Raza, J.-B. Liu, and S. Qu, "On mixed metric dimension of rotationally symmetric graphs," IEEE Access, vol. 8, pp. 11560-11569, 2020.

[30] M. Abas and T. Vetrík, "Metric dimension of Cayley digraphs of split metacyclic groups," Theoretical Computer Science, vol. 809, pp. 61-72, 2020.

[31] T. Vetrik, "On the metric dimension of directed and undirected circulant graphs," Discussiones Mathematicae Graph Theory, vol. 40, no. 1, pp. 67-76, 2020.

[32] A. Simić, M. Bogdanović, and J. Milošević, "The binary locating-dominating number of some convex polytopes," Ars Mathematica Contemporanea, vol. 13, pp. 367-377, 2017.

[33] H. Raza, S. Hayat, and X.-F. Pan, "Binary locating-dominating sets in rotationally-symmetric convex polytopes," Symmetry, vol. 10, no. 12, pp. 727-745, 2018.

[34] A. Savic, Z. Maksimovic, and M. Bogdanovic, "The openlocating-dominating number of some convex polytopes," FILOMAT, vol. 32, no. 2, pp. 635-642, 2018. 
[35] J.-B. Liu, J. Cao, A. Alofi, A. AL-Mazrooei, and A. Elaiw, "Applications of Laplacian spectra for n-prism networks n-Prism Networks," Neurocomputing, vol. 198, pp. 69-73, 2016.

[36] J.-B. Liu and J. Cao, "The resistance distances of electrical networks based on Laplacian generalized inverse," Neurocomputing, vol. 167, pp. 306-313, 2015.

[37] J.-B. Liu, X.-F. Pan, L. Yu, and D. Li, "Complete characterization of bicyclic graphs with minimal Kirchhoff index," Discrete Applied Mathematics, vol. 200, pp. 95-107, 2016.

[38] J.-B. Liu, C. Wang, S. Wang, and B. Wei, "Zagreb indices and multiplicative Zagreb indices of Eulerian graphs," Bulletin of the Malaysian Mathematical Sciences Society, vol. 42, no. 1, pp. 67-78, 2019.

[39] J.-B. Liu, J. Zhao, and Z. Cai, "On the generalized adjacency, Laplacian and signless Laplacian spectra of the weighted edge corona networks," Physica A, vol. 540, p. 12307, 2020.

[40] I. Lukovits, "The detour index," Croatica Chemica Acta, vol. 69 , pp. 873-882, 1996.

[41] I. Lukovits, "Indicators for atoms included in cycles," Journal of Chemical Information and Computer Sciences, vol. 36, no. 1, pp. 65-68, 1996.

[42] N. Trinajstić, S. Nikolić, B. Lučić, D. Amić, and Z. Mihalić, "The detour matrix in chemistry," Journal of Chemical Information and Modeling, vol. 37, pp. 631-638, 1997.

[43] G. Rücker and C. Rücker, "Symmetry-aided computation of the detour matrix and the detour index," Journal of Chemical Information and Computer Sciences, vol. 38, no. 4, pp. 710-714, 1998.

[44] I. Lukovits and M. Razinger, "On calculation of the detour index," Journal of Chemical Information and Computer Sciences, vol. 37, no. 2, pp. 283-286, 1997.

[45] N. Trinajstić, S. Nikolić, and Z. Mihalić, "On computing the molecular detour matrix," International Journal of Quantum Chemistry, vol. 65, pp. 415-419, 1998.

[46] F. Harary, Graph Theory, p. 203, Addison-Wesley, Reading, MA, USA, 1969.

[47] A. Mahmiani, O. Khormali, and A. Iranmanesh, "The edge versions of detour index," MATCH Communications in Mathematical and in Computer Chemistry, vol. 62, no. 2, pp. 419-431, 2009.

[48] B. Zhou and X. Cai, "On detour index," MATCH Communications in Mathematical and in Computer Chemistry, vol. 63, pp. 199-210, 2010.

[49] X. Qi and B. Zhou, "Detour index of a class of unicyclic graphs," Filomat, vol. 24, no. 1, pp. 29-40, 2010.

[50] C. Du, "Minimum detour index of bicyclic graphs, $\mathrm{MATCH}$ Commun," Mathematical and in Computer Chemistry, vol. 68, no. 1, pp. 357-370, 2012.

[51] W. Fang, Z. Q. Cai, and X. X. Li, "Minimum detour index of tricyclic graphs," Journal of Chemistry, vol. 2019, Article ID 6031568, 8 pages, 2019.

[52] A. Karbasioun, A. R. Ashrafi, and M. V. Diudea, "Distance and detour matrices of an infinite class of dendrimer nanostars," MATCH Communications in Mathematical and in Computer Chemistry, vol. 63, no. 1, pp. 239-246, 2010.

[53] R. Wu and H. Deng, "On the detour index of a chain of C20 fullerenes," Journal of Optoelectronic and Biomedical Materials, vol. 8, no. 2, pp. 45-48, 2016.

[54] V. Kaladevi and A. Abinayaa, "On detour distance Laplacian energy," Journal of Informatics and Mathematical Sciences, vol. 9, no. 3, pp. 721-732, 2017.

[55] H. O. Abdullah and Z. I. Omar, "Edge restricted detour index of some graphs," Journal of Discrete Mathematical Sciences and Cryptography, vol. 23, no. 4, pp. 861-877, 2020.
[56] A. A. Dobrynin, R. Entringer, and I. Gutman, "Wiener index of trees: theory and applications," Acta Applicandae Mathematicae, vol. 66, no. 3, pp. 211-249, 2001.

[57] M. Imran, S. A. Ul Haq Bokhary, and A. Q. Baig, "On families of convex polytopes with constant metric dimension," Computers \& Mathematics with Applications, vol. 60, no. 9, pp. 2629-2638, 2010. 